\title{
Synthesis and Antimicrobial Evaluation of Some Novel Trisubstituted s-Triazine Derivatives Based on Isatinimino, Sulphonamido, and Azacarbazole
}

\author{
Sonika Jain, Anamika Sharma, Meenakshi Agrawal, Swapnil Sharma, \\ Jaya Dwivedi, and D. Kishore
}

Department of Chemistry, Banasthali University, Banasthali 304022, India

Correspondence should be addressed to Sonika Jain; sonikajain85@gmail.com

Received 20 December 2011; Accepted 3 May 2012

Academic Editor: Dexin Kong

Copyright (C) 2013 Sonika Jain et al. This is an open access article distributed under the Creative Commons Attribution License, which permits unrestricted use, distribution, and reproduction in any medium, provided the original work is properly cited.

A study directed towards exploring the temperature-dependent reactivity of the chlorine atoms of 2,4,6-trichloro-s-triazine (TCT) in the nucleophilic displacement reaction, allowed a facile replacement of its chlorine atoms in succession with (i) $\mathrm{N}$-amino methyl substituted isatin-3-hydrazones, (ii) $\mathrm{N}_{1}$-substituted-4-amino benzene sulphonamides, and (iii) 8-amino-4oxo-N-benzyl-azacarbazole to produce the corresponding 2,4,6-trisubstituted-s-triazine, namely; 2-(N-amino methyl substituted isatin-3-hydrazinyl)-4-( $\mathrm{N}_{1}$-substituted-4' -amino benzenesulfonamidyl)-6-( $8^{\prime}$-amino- $4^{\prime}$-oxo-N-benzylazacarbazolyl)-1,3,5triazine derivatives in acceptable yields. The compounds prepared were further evaluated for their antibacterial activity against $E$. coli and B. subtilis and antifungal activities against A. niger and A. flavus, and some of them showed promising activity profile.

\section{Introduction}

Due to rapid development in drug resistance, tolerance, and side effects, there is a critical need for the development of a new generation of antimicrobial agents that exhibit improved pharmacological properties and drug-resistance profiles. In this aspect substituted s-triazine derivatives have been received central attention due to their significant antimicrobial [1], antibacterial [2], antifungal [3], anti-HIV [4], anticancer [5], and a wide array of other biological activities $[6,7]$. Our hypothesis is to incorporate the certain pharmacophoric features and scaffolds on the templates of s-triazine molecule in order to design as a novel antimicrobial agents.

Mannich bases of isatin derivatives have been reported to form an integral part of the bioactive molecules exhibiting wide range of activities such as cytotoxic [8], anticonvulsant [9], antidepressant [10], and anti-inflammatory [11] agents. Sulphonamides have been widely known in the literature to exhibit a broad range of biological activities [12, 13]. The $\mathrm{SO}_{2}-\mathrm{NH}$ group of sulphonamide constitutes a key structural motif shared by a large number of bioactive compounds spanning a variety of effects such as the microbial activities [14], specific enzyme inhibition [15] and hormonal regulation [16].

The ubiquitous presence of azacarbazoles and pyridocarbazoles in a vast array of biologically active molecules (such as ellipticine, olivacine and carbaquinacine [17], to name a few) have stimulated as intense research efforts to the synthesis of their structural analogues where different constitution and biological activity of new materials could allow them to be used as novel chemotherapeutic agents.

Greatly encouraged by the biological profiles of these heterocyclic scaffolds, we aimed in the present work to synthesize 2,4,6-trisubstituted-s-triazine molecules incorporating in them the structural features of (i) N-Mannich's bases of isatin-3-hydrazones, 2(a-f), (ii) established sulpha drugs, 4(a,b), (iii) N-benzyl-4-oxo azacarbazole derivatives (6), on the premise that their presence in tandem in a single molecular framework in $7(\mathbf{a}-\mathbf{l})$ should contribute significantly to the biological activity in the resulting molecules and should allow them to be used as novel chemotherapeutic agents. 


\section{Experimental Section}

2.1. General Remarks. Melting points were determined in open glass capillaries and are uncorrected. The reactions were monitored by the TLC on silica gel "G" plates and were visualized with UV light and by exposing to iodine. The purity of the compounds was checked by spectral data, namely, IR, NMR, mass and elemental analysis. IR spectra were recorded on CE (SIMADZU) FTIR-8400S. ${ }^{1} \mathrm{HNMR}$ spectra were recorded on model Avance 300 (Bruker $300 \mathrm{MHz}$ ) using DMSO- $d_{6}$ as solvent and TMS as an internaly reference.

\section{Chemical Synthesis}

\subsection{General Methods for the Preparation of 3(a-f), 5(a-1), 7(a-1)}

3.1.1. Preparation of 3-(2-(4,6-dichloro-1,3,5-triazin-2yl)hydrazono)-1-(morpholinomethyl)indolin-2-one

(3a). Cyanuric chloride (1) ( $0.5 \mathrm{~g}, 0.002$ moles) was taken in dry THF containing $\mathrm{K}_{2} \mathrm{CO}_{3}(0.1 \mathrm{~g})$. 1-(morpholinomethyl)indolin-2-one-3-hydrazone (2a) (0.7 g, 0.002 moles) was added to it. Reaction was kept on stirring at $0-5^{\circ} \mathrm{C}$ for $2-3 \mathrm{hr}$. Reaction mixture was poured in crushed ice and neutralized with dil. $\mathrm{HCl}$. The resulting solid was filtered, washed with dil. Ethanol. The resultant solid was dried and recrystallized from ethanol: water $(1: 9)$ mixture to give $3 \mathbf{a}, 0.48 \mathrm{~g}$ (yield $68.8 \%)$ m.p. $180-181^{\circ}$ C. Anal. Calcd. C, 47.07; H, 3.70; N, 24.02; $\mathrm{Cl}, 17.37\left(\mathrm{C}_{16} \mathrm{H}_{15} \mathrm{Cl}_{2} \mathrm{~N}_{7} \mathrm{O}_{2}\right)$. Found: $\mathrm{C} 47.01 ; \mathrm{H}, 3.75$; $\mathrm{N}, 23.98 ; \mathrm{Cl}, 17.31 \mathrm{IR}(\mathrm{KBr}) \mathrm{cm}^{-1} 3400(\mathrm{NH}), 3100$ (arom. Str.), $1711(\mathrm{C}=\mathrm{O}),{ }^{1} \mathrm{H}-\mathrm{NMR}\left(\mathrm{DMSO}-d_{6}\right)-\delta, 10.7(1 \mathrm{H}, \mathrm{s}$, $\mathrm{NH}), 7.77-6.81(4 \mathrm{H}, \mathrm{m}, \mathrm{Ar}-\mathrm{H}), 4.03\left(2 \mathrm{H}, \mathrm{s}, \mathrm{CH}_{2}\right), 3.65(4 \mathrm{H}$, $\left.\mathrm{t}, \mathrm{CH}_{2}, \mathrm{~J}=1.1 \mathrm{~Hz}\right), 2.47\left(4 \mathrm{H}, \mathrm{t}, \mathrm{CH}_{2}, \mathrm{~J}=1.8 \mathrm{~Hz}\right)$.

Compound $\mathbf{3 ( b - f )}$ was prepared by a similar method using appropriate reactants with required change in stirring time.

3-(2-(4,6-dichloro-1,3,5-triazin-2-yl)hydrazono)-1-(piperidin-1-ylmethyl)indolin-2-one (3b). Yield 70\%; m.p. 205$206^{\circ} \mathrm{C}$; Anal. Calcd. C 50.26; H, 4.22; N, 24.13; Cl, 17.45 $\left(\mathrm{C}_{17} \mathrm{H}_{17} \mathrm{Cl}_{2} \mathrm{~N}_{7} \mathrm{O}\right)$. Found: C 50.20; $\mathrm{H}, 4.02 ; \mathrm{N}, 24.01 ; \mathrm{Cl}$, 17.41; IR (KBr) cm $\mathrm{cm}^{-1} 3390(\mathrm{NH}), 3100$ (arom. Str.), 1675 (C=O), 1602 ( $\mathrm{C}=\mathrm{N}$ str.), 1425 ( $\mathrm{C}=\mathrm{C}$ aromatic str.), ${ }^{1} \mathrm{H}-\mathrm{NMR}$ $\left(\mathrm{DMSO}-d_{6}\right)-\delta, 10.5(1 \mathrm{H}, \mathrm{s}, \mathrm{NH}), 7.86-6.78(4 \mathrm{H}, \mathrm{m}, \mathrm{Ar}-\mathrm{H})$, $4.05\left(2 \mathrm{H}, \mathrm{s}, \mathrm{CH}_{2}\right), 2.45\left(4 \mathrm{H}, \mathrm{d}, \mathrm{CH}_{2}, \mathrm{~J}=1.8 \mathrm{~Hz}\right), 1.59(2 \mathrm{H}, \mathrm{m}$, $\left.\mathrm{CH}_{2}\right), 1.53\left(4 \mathrm{H}, \mathrm{m}, \mathrm{CH}_{2}\right)$.

3-(2-(4,6-dichloro-1,3,5-triazin-2-yl)hydrazono)-1-(pyrrolidin-1-ylmethyl)indolin-2-one (3c). Yield 69\%; m.p. 220$221^{\circ} \mathrm{C}$; Anal. Calcd. C, 48.99; H, 3.85; N, 25.00; Cl, 18.08 $\left(\mathrm{C}_{16} \mathrm{H}_{15} \mathrm{Cl}_{2} \mathrm{~N}_{7} \mathrm{O}\right)$. Found: $\mathrm{C} 48.91 ; \mathrm{H}, 3.81 ; \mathrm{N}, 24.87 ; \mathrm{Cl}, 18.02$ IR $(\mathrm{KBr}) \mathrm{cm}^{-1} 3410(\mathrm{NH}), 1710(\mathrm{C}=\mathrm{O}), 3200$ (aromatic Str.), $1610(\mathrm{C}=\mathrm{N}), 1425(\mathrm{C}=\mathrm{C}),{ }^{1} \mathrm{H}-\mathrm{NMR}\left(\mathrm{DMSO}-d_{6}\right)-\delta, 10.2$ $(1 \mathrm{H}, \mathrm{s}, \mathrm{NH}), 7.67-6.52(4 \mathrm{H}, \mathrm{m}, \mathrm{Ar}-\mathrm{H}), 4.03\left(2 \mathrm{H}, \mathrm{s}, \mathrm{CH}_{2}\right)$, $2.51\left(4 \mathrm{H}, \mathrm{t}, \mathrm{CH}_{2}, \mathrm{~J}=2.2 \mathrm{~Hz}\right), 1.68\left(4 \mathrm{H}, \mathrm{m}, \mathrm{CH}_{2}\right)$.

3-(2-(4,6-dichloro-1,3,5-triazin-2-yl)hydrazono)-1-((4-methylpiperazin-1-yl)methyl)indolin-2-one (3d). Yield 69\%; m.p.
225-226 ${ }^{\circ}$ C; Anal. Calcd. C, 48.47; H, 4.31; N, 26.60; Cl, 16.83 $\left(\mathrm{C}_{17} \mathrm{H}_{18} \mathrm{Cl}_{2} \mathrm{~N}_{8} \mathrm{O}\right)$. Found: $\mathrm{C} 48.41 ; \mathrm{H}, 4.25 ; \mathrm{N}, 26.50 ; \mathrm{Cl}$, 16.72; IR (KBr) cm cm $^{-1} 386(\mathrm{NH}), 3050$ (aromatic Str.), 1700 $(\mathrm{C}=\mathrm{O}), 1602(\mathrm{C}=\mathrm{N}), 1420(\mathrm{C}=\mathrm{C}$ aromatic Str. $),{ }^{1} \mathrm{H}-\mathrm{NMR}$ $\left(\mathrm{DMSO}-d_{6}\right)-\delta 10.8(1 \mathrm{H}, \mathrm{s}, \mathrm{NH}), 7.86-6.45(4 \mathrm{H}, \mathrm{m}, \mathrm{Ar}-\mathrm{H})$, $4.03\left(2 \mathrm{H}, \mathrm{s}, \mathrm{CH}_{2}\right), 2.35\left(8 \mathrm{H}, \mathrm{t}, \mathrm{CH}_{2}, \mathrm{~J}=2.1 \mathrm{~Hz}\right), 2.26(3 \mathrm{H}, \mathrm{s}$, $\left.\mathrm{CH}_{3}\right)$.

3-(2-(4,6-dichloro-1,3,5-triazin-2-yl)hydrazono)-1-((4-ethylpiperazin-1-yl)methyl)indolin-2-one (3e). Yield 71\%; m.p. 232-233 ${ }^{\circ} \mathrm{C}$; Anal. Calcd. C, 49.66; H, 4.63; N, 25.74; Cl, 16.29 $\left(\mathrm{C}_{18} \mathrm{H}_{20} \mathrm{Cl}_{2} \mathrm{~N}_{8} \mathrm{O}\right)$. Found: $\mathrm{C} 49.50 ; \mathrm{H}, 4.55 ; \mathrm{N}, 25.65 ; \mathrm{Cl}$, 16.10; IR (KBr) cm cm $^{-1} 390(\mathrm{NH}), 3050$ (aromatic str.), 1715 $(\mathrm{C}=\mathrm{O}), 1602(\mathrm{C}=\mathrm{N}), 1425\left(\mathrm{C}=\mathrm{C}\right.$ str.), ${ }^{1} \mathrm{H}-\mathrm{NMR}$ (DMSO$\left.d_{6}\right)-\delta 10.7(1 \mathrm{H}, \mathrm{s}, \mathrm{NH}), 7.86-6.91(4 \mathrm{H}, \mathrm{m}, \mathrm{Ar}-\mathrm{H}), 4.03(2 \mathrm{H}$, $\left.\mathrm{s}, \mathrm{CH}_{2}\right), 2.35\left(8 \mathrm{H}, \mathrm{t}, \mathrm{CH}_{2}, \mathrm{~J}=2.4 \mathrm{~Hz}\right), 2.38\left(2 \mathrm{H}, \mathrm{m}, \mathrm{CH}_{2}\right)$, $1.02\left(3 \mathrm{H}, \mathrm{t}, \mathrm{CH}_{3}, \mathrm{~J}=1.2 \mathrm{~Hz}\right)$.

3-(2-(4,6-dichloro-1,3,5-triazin-2-yl)hydrazono)-1-((4-phenylpiperazin-1-yl)methyl)indolin-2-one (3f). Yield 72\%; m.p. 228-229 ${ }^{\circ}$; Anal. Calcd. C, 54.67; H, 4.17; N, 23.18; Cl, $14.67\left(\mathrm{C}_{22} \mathrm{H}_{20} \mathrm{Cl}_{2} \mathrm{~N}_{8} \mathrm{O}\right)$. Found: $\mathrm{C} 54.55 ; \mathrm{H}, 4.07 ; \mathrm{N}, 23.10$; $\mathrm{Cl}, 14.52$; IR $(\mathrm{KBr}) \mathrm{cm}^{-1} 3245(\mathrm{NH}), 3010$ (aromatic Str.), $1720(\mathrm{C}=\mathrm{O}), 1602$ ( $\mathrm{C}=\mathrm{N}$ str.), 1425 ( $\mathrm{C}=\mathrm{C}$ str.), ${ }^{1} \mathrm{H}-\mathrm{NMR}$ $\left(\mathrm{DMSO}-d_{6}\right)-10.8(1 \mathrm{H}, \mathrm{s}, \mathrm{NH}), 7.86-6.79(9 \mathrm{H}, \mathrm{m}, \mathrm{Ar}-\mathrm{H})$, $4.03\left(2 \mathrm{H}, \mathrm{s}, \mathrm{CH}_{2}\right), 3.44\left(4 \mathrm{H}, \mathrm{t}, \mathrm{CH}_{2}, \mathrm{~J}=5.6 \mathrm{~Hz}\right), 2.48(4 \mathrm{H}, \mathrm{t}$, $\mathrm{CH}_{2}, \mathrm{~J}=1.6 \mathrm{~Hz}$ ).

3.1.2. Preparation of 4-(4-chloro-6-(2-(1-(morpholinomethyl)-2-oxoindolin-3-ylidene)hydrazinyl)-1,3,5-triazin-2ylamino)-N-(thiazol-2-yl)benzenesulfonamide 5(a). Compound 3a ( $0.3 \mathrm{~g}, 0.00073$ moles) was taken in dry THF and $\mathrm{K}_{2} \mathrm{CO}_{3}(0.1 \mathrm{~g})$. Sulphathiazole $4 \mathrm{a}(0.187 \mathrm{~g}, 0.00073$ moles $)$ was added to this. Reaction mixture was kept on stirring at $30-35^{\circ} \mathrm{C}$ for $2-3 \mathrm{hr}$ and was then poured in to crushed ice and neutralized with dil. $\mathrm{HCl}$. The resulting solid was filtered, washed with dil. Ethanol. The resultant solid was dried and recrystallized from ethanol: water $(1: 9)$ mixture to give 5a, $0.182 \mathrm{~g}$ (yield 58.8\%) m.p. 200-201 ${ }^{\circ}$ C. Anal. Calcd. C, 63.41; $\mathrm{H}, 4.90 ; \mathrm{N}, 21.75 ; \mathrm{S}, 3.32\left(\mathrm{C}_{51} \mathrm{H}_{47} \mathrm{~N}_{15} \mathrm{O}_{4} \mathrm{~S}\right)$. Found: $\mathrm{C} 63.01$; $\mathrm{H}, 4.75$; N, 21.55; S, 3.02; IR (KBr) cm $3300(\mathrm{NH}), 3011$ (aromatic Str.), 1695 (C=O), 1602 (C=N str.), 1425 (C=C str.), 1360,1162 ( $\mathrm{S}=\mathrm{O}),{ }^{1} \mathrm{H}-\mathrm{NMR}$ (DMSO-d $\left.{ }_{6}\right)-12.64(1 \mathrm{H}, \mathrm{s}$, $\mathrm{NH}), 10.67$ (1H, s,NH), $9.44(1 \mathrm{H}, \mathrm{s}, \mathrm{NH}), 7.61-7.18(9 \mathrm{H}, \mathrm{m}$, $\mathrm{Ar}-\mathrm{H},), 3.87(1 \mathrm{H}, \mathrm{t}, \mathrm{CH}, \mathrm{J}=0.9 \mathrm{~Hz}), 3.65\left(4 \mathrm{H}, \mathrm{q}, \mathrm{CH}_{2}\right), 3.11$ $\left(2 \mathrm{H}, \mathrm{s}, \mathrm{CH}_{2}\right), 2.67\left(4 \mathrm{H}, \mathrm{q}, \mathrm{CH}_{2}\right)$.

Compound $\mathbf{5}(\mathbf{b}-\mathbf{l})$ were prepared by a similar method using appropriate reactants with required change in temperature and stirring time.

4-(4-chloro-6-(2-(1-(morpholinomethyl)-2-oxoindolin-3-ylidene)hydrazinyl)-1,3,5-triazin-2-ylamino)-N-(4-methylpyrimidin-2-yl)benzenesulfonamide (5b). Yield 67\%; m.p. 221-222 ${ }^{\circ}$ C; Anal. Calcd. C, 63.41; H, 4.90; N, 21.75; S, 3.32 $\left(\mathrm{C}_{51} \mathrm{H}_{47} \mathrm{~N}_{15} \mathrm{O}_{4} \mathrm{~S}\right)$. Found: $\mathrm{C}$ 63.01; H, 4.75; N, 21.55; S, 3.02; IR $(\mathrm{KBr}) \mathrm{cm}^{-1} 3337(\mathrm{NH}), 3015$ (aromatic str.), $1710(\mathrm{C}=\mathrm{O})$, $1587(\mathrm{C}=\mathrm{N}), 1450$ (C=C str.), 1311, $1134(\mathrm{~S}=\mathrm{O}),{ }^{1} \mathrm{H}-\mathrm{NMR}$ $\left(\mathrm{DMSO}-d_{6}\right)-11.22(1 \mathrm{H}, \mathrm{s}, \mathrm{NH}), 10.79(1 \mathrm{H}, \mathrm{s}, \mathrm{NH}), 9.44(1 \mathrm{H}$, 
s, NH), 7.35-6.93(10H, m, Ar-H), 3.65(4H, t, $\mathrm{CH}_{2}, \mathrm{~J}=$ $1.3 \mathrm{~Hz}), 2.67(4 \mathrm{H}, \mathrm{q}, \mathrm{CH}), 2.33\left(3 \mathrm{H}, \mathrm{s}, \mathrm{CH}_{3}\right)$.

4-(4-chloro-6-(2-(2-oxo-1-(piperidin-1-ylmethyl)indolin-3ylidene)hydrazinyl)-1,3,5-triazin-2-ylamino)- $N$-(thiazol-2yl)benzenesulfonamide (5c). Yield $68 \%$; m.p. $299-300^{\circ} \mathrm{C}$; Anal. Calcd. C, 63.41; H, 4.90; N, 21.75; S, 3.32 $\left(\mathrm{C}_{51} \mathrm{H}_{47} \mathrm{~N}_{15} \mathrm{O}_{4} \mathrm{~S}\right)$. Found: $\mathrm{C}$ 63.01; H, 4.75; N, 21.55; S, 3.02; IR (KBr) $\mathrm{cm}^{-1} 3180(\mathrm{NH}), 3020$ (aromatic Str.), 1712 $(\mathrm{C}=\mathrm{O}), 1590(\mathrm{C}=\mathrm{N}), 1480(\mathrm{C}=\mathrm{C}$ str. $), 1357,1134(\mathrm{~S}=\mathrm{O})$, ${ }^{1} \mathrm{H}-\mathrm{NMR}\left(\mathrm{DMSO}-d_{6}\right)-12.64(1 \mathrm{H}, \mathrm{s}, \mathrm{NH}), 10.67(1 \mathrm{H}, \mathrm{s}$, $\mathrm{NH}), 9.44(1 \mathrm{H}, \mathrm{s}, \mathrm{NH}), 7.86-6.75(10 \mathrm{H}, \mathrm{m}, \mathrm{Ar}-\mathrm{H}), 4.03(2 \mathrm{H}$, s, $\left.\mathrm{CH}_{2}\right), 2.45\left(4 \mathrm{H}, \mathrm{t}, \mathrm{CH}_{2}, \mathrm{~J}=0.9 \mathrm{~Hz}\right), 1.59\left(2 \mathrm{H}, \mathrm{q}, \mathrm{CH}_{2}\right), 1.53$ $\left(4 \mathrm{H}, \mathrm{m}, \mathrm{CH}_{2}\right)$.

4-(4-chloro-6-(2-(2-oxo-1-(piperidin-1-ylmethyl)indolin-3ylidene)hydrazinyl)-1,3,5-triazin-2-ylamino)- $N$-(4-methylpyrimidin-2-yl)benzenesulfonamide (5d). Yield 69\%; m.p. 291-292 ${ }^{\circ}$ C; Anal. Calcd. C, 63.41; H, 4.90; N, 21.75; S, 3.32 $\left(\mathrm{C}_{51} \mathrm{H}_{47} \mathrm{~N}_{15} \mathrm{O}_{4} \mathrm{~S}\right)$. Found: $\mathrm{C} 63.01 ; \mathrm{H}, 4.75 ; \mathrm{N}, 21.55 ; \mathrm{S}, 3.02$; IR (KBr) cm ${ }^{-1} 3180(\mathrm{NH}), 3025$ (aromatic str.), $1712(\mathrm{C}=\mathrm{O})$, $1595(\mathrm{C}=\mathrm{N}), 1490$ (C=C str.), 1357, $1134(\mathrm{~S}=\mathrm{O}),{ }^{1} \mathrm{H}-\mathrm{NMR}$ $\left(\mathrm{DMSO}_{-} d_{6}\right)-11.27(1 \mathrm{H}, \mathrm{s}, \mathrm{NH}), 10.67(1 \mathrm{H}, \mathrm{s}, \mathrm{NH}), 9.44$ $(1 \mathrm{H}, \mathrm{s}, \mathrm{NH}), 8.11(1 \mathrm{H}, \mathrm{s}, \mathrm{Ar}-\mathrm{H}), 7.86-7.24(9 \mathrm{H}, \mathrm{m}, \mathrm{Ar}-\mathrm{H})$, $4.03\left(2 \mathrm{H}, \mathrm{s}, \mathrm{CH}_{2}\right), 2.45\left(4 \mathrm{H}, \mathrm{t}, \mathrm{CH}_{2}, \mathrm{~J}=0.78 \mathrm{~Hz}\right), 2.33(3 \mathrm{H}, \mathrm{s}$, $\left.\mathrm{CH}_{3}\right), 1.59\left(2 \mathrm{H}, \mathrm{q}, \mathrm{CH}_{2}\right), 1.53\left(4 \mathrm{H}, \mathrm{m}, \mathrm{CH}_{2}\right)$.

4-(4-chloro-6-(2-(2-oxo-1-(pyrrolidin-1-ylmethyl)indolin-3ylidene)hydrazinyl)-1,3,5-triazin-2-ylamino)- $N$-(thiazol-2yl)benzenesulfonamide (5e). Yield 70\%; m.p. $240-241^{\circ} \mathrm{C}$; Anal. Calcd. C, 63.41; H, 4.90; N, 21.75; S, $3.32\left(\mathrm{C}_{51} \mathrm{H}_{47} \mathrm{~N}_{15} \mathrm{O}_{4} \mathrm{~S}\right)$. Found: C 63.01; H, 4.75; N, 21.55; S, 3.02; IR $(\mathrm{KBr}) \mathrm{cm}^{-1}$ $3180(\mathrm{NH}), 3125$ (aromatic str.), $1712(\mathrm{C}=\mathrm{O}), 1480(\mathrm{C}=\mathrm{N}$ str.), 1505 (C=C str.), 1357, $1134(\mathrm{~S}=\mathrm{O}),{ }^{1} \mathrm{H}-\mathrm{NMR}$ (DMSO$\left.d_{6}\right)-12.64(1 \mathrm{H}, \mathrm{s}, \mathrm{NH}), 10.67(1 \mathrm{H}, \mathrm{s}, \mathrm{NH}), 9.44(1 \mathrm{H}, \mathrm{s}, \mathrm{NH})$, 7.86-6.75 (10H, m, Ar-H), $4.03\left(2 \mathrm{H}, \mathrm{s}, \mathrm{CH}_{2}\right), 2.51(4 \mathrm{H}, \mathrm{t}$, $\left.\mathrm{CH}_{2}, \mathrm{~J}=5.6 \mathrm{~Hz}\right), 1.68\left(4 \mathrm{H}, \mathrm{m}, \mathrm{CH}_{2}\right)$.

4-(4-chloro-6-(2-(2-oxo-1-(pyrrolidin-1-ylmethyl)indolin3-ylidene)hydrazinyl)-1,3,5-triazin-2-ylamino)- $\mathrm{N}$-(4-methylpyrimidin-2-yl)benzenesulfonamide (5f). Yield 65\%; m.p. 250-251 ${ }^{\circ} \mathrm{C}$; Anal. Calcd. C, 63.41; H, 4.90; N, 21.75; S, 3.32 $\left(\mathrm{C}_{51} \mathrm{H}_{47} \mathrm{~N}_{15} \mathrm{O}_{4} \mathrm{~S}\right)$. Found: $\mathrm{C}$ 63.01; H, 4.75; N, 21.55; S, 3.02; IR (KBr) cm $\mathrm{cm}^{-1} 3225(\mathrm{NH}), 3120$ (aromatic str.), 1715 $(\mathrm{C}=\mathrm{O}), 1485$ ( $\mathrm{C}=\mathrm{N}$ str.), 1510 ( $\mathrm{C}=\mathrm{C}$ str.), 1357, $1134(\mathrm{~S}=\mathrm{O})$, ${ }^{1}$ H-NMR (DMSO- $\left.d_{6}\right)-11.27(1 \mathrm{H}, \mathrm{s}, \mathrm{NH}), 10.67(1 \mathrm{H}, \mathrm{s}$, $\mathrm{NH}), 9.44(1 \mathrm{H}, \mathrm{s}, \mathrm{NH}), 8.11(1 \mathrm{H}, \mathrm{s}, \mathrm{Ar}-\mathrm{H}), 7.86-7.24(9 \mathrm{H}, \mathrm{m}$, $\mathrm{Ar}-\mathrm{H}), 4.03\left(2 \mathrm{H}, \mathrm{s}, \mathrm{CH}_{2}\right), 2.51\left(4 \mathrm{H}, \mathrm{t}, \mathrm{CH}_{2}, \mathrm{~J}=0.9 \mathrm{~Hz}\right), 2.33$ $\left(3 \mathrm{H}, \mathrm{s}, \mathrm{CH}_{3}\right), 1.68\left(4 \mathrm{H}, \mathrm{m}, \mathrm{CH}_{2}\right)$.

4-(4-chloro-6-(2-(1-((4-methylpiperazin-1-yl)methyl)-2-oxoindolin-3-ylidene)hydrazinyl)-1,3,5-triazin-2-ylamino)- $N$ (thiazol-2-yl)benzenesulfonamide (5g). Yield 65\%; m.p. 233-234 ${ }^{\circ}$ C; Anal. Calcd. C, 63.41; H, 4.90; N, 21.75; S, 3.32 $\left(\mathrm{C}_{51} \mathrm{H}_{47} \mathrm{~N}_{15} \mathrm{O}_{4} \mathrm{~S}\right)$. Found: $\mathrm{C}$ 63.01; H, 4.75; N, 21.55; S, 3.02; IR (KBr) cm $\mathrm{cm}^{-1} 3195(\mathrm{NH}), 3230$ (aromatic str.), 1712 $(\mathrm{C}=\mathrm{O}), 1490$ (C=N str.), 1525 (C=C str.), 1357, $1134(\mathrm{~S}=\mathrm{O})$, ${ }^{1} \mathrm{H}-\mathrm{NMR}\left(\mathrm{DMSO}-d_{6}\right)-10.67(1 \mathrm{H}, \mathrm{s}, \mathrm{NH}), 10.04(1 \mathrm{H}, \mathrm{s}$,
$\mathrm{NH}), 9.44(1 \mathrm{H}, \mathrm{s}, \mathrm{NH}), 7.86-6.38(10 \mathrm{H}, \mathrm{m}, \mathrm{Ar}-\mathrm{H}), 4.03(2 \mathrm{H}$, $\left.\mathrm{s}, \mathrm{CH}_{2}\right), 2.35\left(8 \mathrm{H}, \mathrm{t}, \mathrm{CH}_{2}, \mathrm{~J}=0.88 \mathrm{~Hz}\right), 2.26\left(3 \mathrm{H}, \mathrm{s}, \mathrm{CH}_{3}\right)$.

4-(4-chloro-6-(2-(1-((4-methylpiperazin-1-yl)methyl)-2oxoindolin-3-ylidene)hydrazinyl)-1,3,5-triazin-2-ylamino)$\mathrm{N}$-(4-methylpyrimidin-2-yl)benzenesulfonamide (5h). Yield 62\%; m.p. 245-246 ${ }^{\circ}$; Anal. Calcd. C, 63.41; H, 4.90; N, 21.75; S, $3.32\left(\mathrm{C}_{51} \mathrm{H}_{47} \mathrm{~N}_{15} \mathrm{O}_{4} \mathrm{~S}\right)$. Found: $\mathrm{C}$ 63.01; H, 4.75; $\mathrm{N}, 21.55$; S, 3.02; IR $(\mathrm{KBr}) \mathrm{cm}^{-1} 3210(\mathrm{NH}), 3235$ (aromatic str.), $1712(\mathrm{C}=\mathrm{O}), 1500(\mathrm{C}=\mathrm{N}$ str. $), 1520(\mathrm{C}=\mathrm{C}$ str. $)$, $1357,1134(\mathrm{~S}=\mathrm{O}),{ }^{1} \mathrm{H}-\mathrm{NMR}\left(\mathrm{DMSO}-d_{6}\right)-11.27(1 \mathrm{H}, \mathrm{s}$, $\mathrm{NH}), 10.67(1 \mathrm{H}, \mathrm{s}, \mathrm{NH}), 9.44(1 \mathrm{H}, \mathrm{s}, \mathrm{NH}), 8.11(1 \mathrm{H}, \mathrm{s}$, $\mathrm{Ar}-\mathrm{H}), 7.86-7.24(9 \mathrm{H}, \mathrm{m}, \mathrm{Ar}-\mathrm{H}), 4.03\left(2 \mathrm{H}, \mathrm{s}, \mathrm{CH}_{2}\right), 2.35$ $\left(8 \mathrm{H}, \mathrm{t}, \mathrm{CH}_{2}, \mathrm{~J}=0.85 \mathrm{~Hz}\right), 2.33\left(3 \mathrm{H}, \mathrm{s}, \mathrm{CH}_{3}\right), 2.26(3 \mathrm{H}, \mathrm{s}$, $\left.\mathrm{CH}_{3}\right)$.

4-(4-chloro-6-(2-(1-((4-ethylpiperazin-1-yl)methyl)-2-oxoindolin-3-ylidene)hydrazinyl)-1,3,5-triazin-2-ylamino)- $N$ (thiazol-2-yl)benzenesulfonamide (5i). Yield 65\%; m.p. 234$235^{\circ} \mathrm{C}$; Anal. Calcd. C, 63.41; H, 4.90; N, 21.75; S, 3.32 $\left(\mathrm{C}_{51} \mathrm{H}_{47} \mathrm{~N}_{15} \mathrm{O}_{4} \mathrm{~S}\right)$. Found: C 63.01; H, 4.75; N, 21.55; S, 3.02; IR $(\mathrm{KBr}) \mathrm{cm}^{-1} 3210(\mathrm{NH}), 3235$ (aromatic str.), $1712(\mathrm{C}=\mathrm{O})$, 1500 (C=N str.), 1520 (C=C str.), 1357,1134 ( $\mathrm{S}=\mathrm{O}),{ }^{1} \mathrm{H}-\mathrm{NMR}$ $\left(\mathrm{DMSO}-d_{6}\right)-12.64(1 \mathrm{H}, \mathrm{s}, \mathrm{NH}), 10.67(1 \mathrm{H}, \mathrm{s}, \mathrm{NH}), 9.44$ $(1 \mathrm{H}, \mathrm{s}, \mathrm{NH}), 7.86-6.75(10 \mathrm{H}, \mathrm{m}, \mathrm{Ar}-\mathrm{H}), 4.03\left(2 \mathrm{H}, \mathrm{s}, \mathrm{CH}_{2}\right)$, $2.38\left(2 \mathrm{H}, \mathrm{q}, \mathrm{CH}_{2}\right), 2.35\left(8 \mathrm{H}, \mathrm{t}, \mathrm{CH}_{2}, \mathrm{~J}=2.1 \mathrm{~Hz}\right), 1.02(3 \mathrm{H}, \mathrm{t}$, $\mathrm{CH}_{3}, \mathrm{~J}=2.09 \mathrm{~Hz}$ ).

4-(4-chloro-6-(2-(1-((4-ethylpiperazin-1-yl)methyl)-2-oxoindolin-3-ylidene)hydrazinyl)-1,3,5-triazin-2-ylamino)- $\mathrm{N}$-(4methylpyrimidin-2-yl)benzenesulfonamide (5j). Yield 71\%; m.p. 255-256 ${ }^{\circ}$; Anal. Calcd. C, 63.41; H, 4.90; N, 21.75; S, $3.32\left(\mathrm{C}_{51} \mathrm{H}_{47} \mathrm{~N}_{15} \mathrm{O}_{4} \mathrm{~S}\right)$. Found: $\mathrm{C} 63.01 ; \mathrm{H}, 4.75 ; \mathrm{N}, 21.55 ; \mathrm{S}$, 3.02; IR (KBr) cm $3115(\mathrm{NH}), 3225$ (aromatic str.), 1712 $(\mathrm{C}=\mathrm{O}), 1510$ ( $\mathrm{C}=\mathrm{N}$ str.), 1525(C=C str.), 1357, $1134(\mathrm{~S}=\mathrm{O})$, ${ }^{1} \mathrm{H}-\mathrm{NMR}$ (DMSO- $\left.d_{6}\right)-11.27(1 \mathrm{H}, \mathrm{s}, \mathrm{NH}), 10.67(1 \mathrm{H}, \mathrm{s}$, $\mathrm{NH}), 9.44(1 \mathrm{H}, \mathrm{s}, \mathrm{NH}), 8.11(1 \mathrm{H}, \mathrm{s}, \mathrm{Ar}-\mathrm{H}), 7.86-7.24(9 \mathrm{H}$, m, Ar-H), $4.03\left(2 \mathrm{H}, \mathrm{s}, \mathrm{CH}_{2}\right), 2.38\left(2 \mathrm{H}, \mathrm{q}, \mathrm{CH}_{2}\right), 2.35(8 \mathrm{H}$, $\left.\mathrm{t}, \mathrm{CH}_{2}, \mathrm{~J}=2.3 \mathrm{~Hz}\right), 2.33\left(3 \mathrm{H}, \mathrm{s}, \mathrm{CH}_{3}\right), 1.02\left(3 \mathrm{H}, \mathrm{t}, \mathrm{CH}_{3}, \mathrm{~J}=\right.$ $2.4 \mathrm{~Hz})$.

4-(4-chloro-6-(2-(2-oxo-1-((4-phenylpiperazin-1-yl)meth$y$ l)indolin-3-ylidene)hydrazinyl)-1,3,5-triazin-2-ylamino)- $N$ (thiazol-2-yl)benzenesulfonamide (5k). Yield 65\%; m.p. 245-246 ${ }^{\circ}$ C; Anal. Calcd. C, 63.41; H, 4.90; N, 21.75; S, 3.32 $\left(\mathrm{C}_{51} \mathrm{H}_{47} \mathrm{~N}_{15} \mathrm{O}_{4} \mathrm{~S}\right)$. Found: $\mathrm{C}$ 63.01; $\mathrm{H}, 4.75 ; \mathrm{N}, 21.55 ; \mathrm{S}$, 3.02; IR (KBr) $\mathrm{cm}^{-1} 3150(\mathrm{NH}), 3225$ (aromatic str.), 1712 $(\mathrm{C}=\mathrm{O}), 1540$ (C=N str.), 1560 (C=C str.), 1357, $1134(\mathrm{~S}=\mathrm{O})$, ${ }^{1} \mathrm{H}-\mathrm{NMR}\left(\mathrm{DMSO}-d_{6}\right)-12.64(1 \mathrm{H}, \mathrm{s}, \mathrm{NH}), 10.67(1 \mathrm{H}, \mathrm{s}$, $\mathrm{NH}), 9.44(1 \mathrm{H}, \mathrm{s}, \mathrm{NH}), 7.86-6.75(14 \mathrm{H}, \mathrm{m}, \mathrm{Ar}-\mathrm{H}), 4.03(2 \mathrm{H}$, s, $\left.\mathrm{CH}_{2}\right), 3.44\left(4 \mathrm{H}, \mathrm{t}, \mathrm{CH}_{2}, \mathrm{~J}=1.2 \mathrm{~Hz}\right), 2.48\left(4 \mathrm{H}, \mathrm{t}, \mathrm{CH}_{2}, \mathrm{~J}=\right.$ $0.8 \mathrm{~Hz})$.

4-(4-chloro-6-(2-(2-oxo-1-((4-phenylpiperazin-1-yl)methyl)indolin-3-ylidene)hydrazinyl)-1,3,5-triazin-2-ylamino)- $N$ (4-methylpyrimidin-2-yl)benzenesulfonamide (51). Yield 60\%; m.p. 234-235 ${ }^{\circ}$; Anal. Calcd. C, 63.41; H, 4.90; N, 21.75; S, $3.32\left(\mathrm{C}_{51} \mathrm{H}_{47} \mathrm{~N}_{15} \mathrm{O}_{4} \mathrm{~S}\right)$. Found: $\mathrm{C}$ 63.01; H, 4.75; N, 21.55; $\mathrm{S}$, 3.02; IR (KBr) cm $3180(\mathrm{NH}), 3235$ (aromatic str.), 1712 
(C=O), 1545 ( $\mathrm{C}=\mathrm{N}$ str.), 1565 ( $\mathrm{C}=\mathrm{C}$ str.), 1357, $1134(\mathrm{~S}=\mathrm{O})$, ${ }^{1} \mathrm{H}-\mathrm{NMR}\left(\mathrm{DMSO}-d_{6}\right)-11.27(1 \mathrm{H}, \mathrm{s}, \mathrm{NH}), 10.67(1 \mathrm{H}, \mathrm{s}$, $\mathrm{NH}), 9.44$ (1H, s, NH), $8.11(1 \mathrm{H}, \mathrm{s}, \mathrm{Ar}-\mathrm{H}), 7.86-6.79(14 \mathrm{H}$, $\mathrm{m}, \mathrm{Ar}-\mathrm{H}), 4.03\left(2 \mathrm{H}, \mathrm{s}, \mathrm{CH}_{2}, \mathrm{~J}=1.1 \mathrm{~Hz}\right), 3.44\left(4 \mathrm{H}, \mathrm{t}, \mathrm{CH}_{2}, \mathrm{~J}=\right.$ $0.9 \mathrm{~Hz}), 2.48\left(4 \mathrm{H}, \mathrm{t}, \mathrm{CH}_{2}, \mathrm{~J}=0.92 \mathrm{~Hz}\right), 2.33\left(3 \mathrm{H}, \mathrm{s}, \mathrm{CH}_{3}\right)$.

3.1.3. Preparation of N-benzyl-8-amino-azacarbazole-4-one (6). A solution of $p$-aminoacetanilide $(0.76 \mathrm{~g}, 0.050 \mathrm{~mol})$ in aqueous $\mathrm{HCl}$ ( $2 \mathrm{~mL}$ conc. $\mathrm{HCl}$ in $5 \mathrm{~mL}$ water) was treated with a cold saturated solution of sodium nitrite $(0.7 \mathrm{gm}$ in $5 \mathrm{~mL}$ water). The solution was kept aside for $10 \mathrm{~min}$. It was then added in portion wise to an ice cooled mixture containing 1benzyl-3-(hydroxymethylidine)-piperidin-4-one (9) (1.26 g, 0.05 mole), sodium acetate trihydrate $(1.78 \mathrm{~g})$, methanol $(10 \mathrm{~mL})$, and water $(20 \mathrm{~mL})$ over a period of 30 minutes with stirring. The contents were allowed to stand for further 30 minutes and the resulting solid was filtered, washed with water, dried, and recrystallised from ethanol. The solid was refluxed with acetic acid $(5 \mathrm{~mL})$ and $\mathrm{HCl}(1 \mathrm{~mL})$ to get the cyclized product (10). The contents were then cooled and poured into cold water with stirring. The separated brown solid was purified by Silica column chromatography (benzene/pet ether, 50/50) to yield cyclized intermediate. The cyclized intermediaye was hydrolysed by heating it in aq. $\mathrm{HCl}$ $(5 \mathrm{~mL})$ for $30 \mathrm{~min}$ to yield 6. M.P. $305^{\circ} \mathrm{C}$.

3.1.4. Preparation of 4-(4-(1-benzyl-4-oxo-2,3,4,5-tetrahydro1H-pyrido [3,2-b] indol-7-ylamino)-6-(2-(1-(morpholinomethyl)-2-oxoindolin-3-ylidene)hydrazinyl)-1,3,5-triazin-2ylamino)-N-(thiazol-2-yl)benzenesulfonamide 7(a). Compound $5 \mathrm{a}(0.1 \mathrm{~g}, 0.00015$ moles $)$ was taken in dry THF and $\mathrm{K}_{2} \mathrm{CO}_{3}$ (0.1 g). N-benzyl-8-amino-azacarbazole-4-one (6) ( $0.046 \mathrm{~g}, 0.00015$ moles) was added to this. Reaction mixture was refluxed at $60-65^{\circ} \mathrm{C}$ for $6 \mathrm{hrs}$ and was then poured in to crushed ice and neutralized with dil. $\mathrm{HCl}$. The resulting solid was filtered, washed with dil. Ethanol. The resultant solid was dried and recrystallized from ethanol: water (1:9) mixture to give 7a, $0.05 \mathrm{~g}$ (yield $48.8 \%$ ) m.p. $249-250^{\circ} \mathrm{C}$. Anal. Calcd. C, 58.56; H, 4.46; N, 20.65; S, $7.27\left(\mathrm{C}_{43} \mathrm{H}_{39} \mathrm{~N}_{13} \mathrm{O}_{5} \mathrm{~S}_{2}\right)$. Found: C, 58.50; H, 4.33; N, 20.55; S, 7.21; IR (KBr) cm $\mathrm{cm}^{-1} 3300(\mathrm{NH}$ symm. str.), 3050 (aromatic str.), $1710(\mathrm{C}=\mathrm{O}), 1602(\mathrm{C}=\mathrm{N}$ str.), ${ }^{1} \mathrm{H}-\mathrm{NMR}$ (DMSO-d $\left.{ }_{6}\right)-\delta, 12.04(1 \mathrm{H}, \mathrm{s}, \mathrm{NH}), 11.62$ $(1 \mathrm{H}, \mathrm{s}, \mathrm{NH}), 10.04(1 \mathrm{H}, \mathrm{s}, \mathrm{NH}), 9.44(1 \mathrm{H}, \mathrm{s}, \mathrm{NH}), 8.91(1 \mathrm{H}$, s, NH), 7.81-6.38 (16H, m, Ar-H), $4.51\left(2 \mathrm{H}, \mathrm{s}, \mathrm{CH}_{2}\right), 4.03$ $\left(2 \mathrm{H}, \mathrm{s}, \mathrm{CH}_{2}\right), 3.65\left(4 \mathrm{H}, \mathrm{t}, \mathrm{CH}_{2}, \mathrm{~J}=5.1 \mathrm{~Hz}\right), 3.39\left(2 \mathrm{H}, \mathrm{t}, \mathrm{CH}_{2}\right.$, $\mathrm{J}=3.6 \mathrm{~Hz}), 2.63\left(2 \mathrm{H}, \mathrm{t}, \mathrm{CH}_{2}, \mathrm{~J}=5.2 \mathrm{~Hz}\right), 2.50\left(4 \mathrm{H}, \mathrm{t}, \mathrm{CH}_{2}\right.$, $\mathrm{J}=4.6 \mathrm{~Hz}$ ).

Compound $7(\mathbf{b}-\mathbf{l})$ were prepared by a similar method using appropriate reactants.

4-(4-(1-benzyl-4-oxo-2,3,4,5-tetrahydro-1H-pyrido [3,2-b] indol-7-ylamino)-6-(2-(1-(morpholinomethyl)-2-oxoindolin3-ylidene)hydrazinyl)-1,3,5-triazin-2-ylamino)-N-(6-methylpyrazin-2yl) benzenesulfonamide (7b). Yield 56\%; m.p. $320^{\circ} \mathrm{C}$; Anal. Calcd. C, 60.66; H, 4.75; N, 22.01; S, 3.60 $\left(\mathrm{C}_{45} \mathrm{H}_{42} \mathrm{~N}_{14} \mathrm{O}_{5} \mathrm{~S}\right)$. Found: C, 60.40; H, 4.65; N, 21.97; S, 3.56; IR (KBr) cm 3300 (NH symm. str.), 3050 (aromatic str.), $1650(\mathrm{C}=\mathrm{O}), 1612$ (C=N str.), ${ }^{1} \mathrm{H}-\mathrm{NMR}\left(\mathrm{DMSO}-d_{6}\right)-\delta$,
$11.63(1 \mathrm{H}, \mathrm{s}, \mathrm{NH}), 10.64(1 \mathrm{H}, \mathrm{s}, \mathrm{NH}), 10.04(1 \mathrm{H}, \mathrm{s}, \mathrm{NH})$, $9.44(1 \mathrm{H}, \mathrm{s}, \mathrm{NH}), 8.91(1 \mathrm{H}, \mathrm{s}, \mathrm{NH}), 8.6-6.38(18 \mathrm{H}, \mathrm{m}$, Ar- $\mathrm{H}), 4.51\left(2 \mathrm{H}, \mathrm{s}, \mathrm{CH}_{2}\right), 4.03\left(2 \mathrm{H}, \mathrm{s}, \mathrm{CH}_{2}\right), 3.65(4 \mathrm{H}, \mathrm{t}$, $\left.\mathrm{CH}_{2}, \mathrm{~J}=6.6 \mathrm{~Hz}\right), 3.39\left(2 \mathrm{H}, \mathrm{t}, \mathrm{CH}_{2}, \mathrm{~J}=5.9 \mathrm{~Hz}\right), 2.63(2 \mathrm{H}, \mathrm{t}$, $\left.\mathrm{CH}_{2}, \mathrm{~J}=2.2 \mathrm{~Hz}\right), 2.50\left(4 \mathrm{H}, \mathrm{t}, \mathrm{CH}_{2}, \mathrm{~J}=3.6 \mathrm{~Hz}\right), 2.33(3 \mathrm{H}, \mathrm{s}$, $\mathrm{CH}_{3}$ ).

4-(4-(1-benzyl-4-oxo-2,3,4,5-tetrahydro-1H-pyrido [3,2-b] indol-7-ylamino)-6-(2-(2-oxo-1-(piperidine-1-ylmethyl)indolin-3-ylidene)hydrazinyl)-1,3,5-triazin-2-ylamino)-N-(thiazol-2-yl)benzenesulfonamide (7c). Yield 62\%; m.p. $315^{\circ} \mathrm{C}$; Anal. Calcd. C, 60.05; H, 4.70; N, 20.69; S, 7.29 $\left(\mathrm{C}_{44} \mathrm{H}_{41} \mathrm{~N}_{13} \mathrm{O}_{4} \mathrm{~S}_{2}\right)$. Found: $\mathrm{C}, 60.50 ; \mathrm{H}, 4.20 ; \mathrm{N}, 20.47$; $\mathrm{S}, 7.22$; IR ( $\mathrm{KBr}) \mathrm{cm}^{-1} 3100$ (NH symm. str.), 3050 (aromatic str.), $1610(\mathrm{C}=\mathrm{O}), 1610$ ( $\mathrm{C}=\mathrm{N}$ str.), ${ }^{1} \mathrm{H}-\mathrm{NMR}$ (DMSO$\left.d_{6}\right)-\delta, 11.53(1 \mathrm{H}, \mathrm{s}, \mathrm{NH}), 10.67(1 \mathrm{H}, \mathrm{s}, \mathrm{NH}), 10.04(1 \mathrm{H}, \mathrm{s}$, $\mathrm{NH}), 9.44(1 \mathrm{H}, \mathrm{s}, \mathrm{NH}), 8.91(1 \mathrm{H}, \mathrm{s}, \mathrm{NH}), 7.86-6.38(17 \mathrm{H}$, $\mathrm{m}, \mathrm{Ar}-\mathrm{H}), 4.51\left(2 \mathrm{H}, \mathrm{s}, \mathrm{CH}_{2}\right), 4.03\left(2 \mathrm{H}, \mathrm{s}, \mathrm{CH}_{2}\right), 3.39(2 \mathrm{H}$, $\left.\mathrm{t}, \mathrm{CH}_{2}, \mathrm{~J}=3.6 \mathrm{~Hz}\right), 2.63\left(2 \mathrm{H}, \mathrm{t}, \mathrm{CH}_{2}, \mathrm{~J}=2.6 \mathrm{~Hz}\right), 2.50$ $\left(4 \mathrm{H}, \mathrm{t}, \mathrm{CH}_{2}, \mathrm{~J}=5.2 \mathrm{~Hz}\right), 1.59\left(2 \mathrm{H}, \mathrm{m}, \mathrm{CH}_{2}\right), 1.53(4 \mathrm{H}, \mathrm{m}$, $\left.\mathrm{CH}_{2}\right)$.

4-(4-(1-benzyl-4-oxo-2,3,4,5-tetrahydro-1H-pyrido [3,2-b] indol-7-ylamino)-6-(2-(2-oxo-1-(piperidine-1-ylmethyl)indolin-3-ylidene)hydrazinyl)-1,3,5-triazin-2-ylamino)-N-(6methylpyrazin-2-yl)benzenesulfonamide (7d). Yield 57\%; m.p. $340^{\circ}$ C; Anal. Calcd. C, 62.15; H, 4.99; N, 22.06; S, 3.61 $\left(\mathrm{C}_{46} \mathrm{H}_{44} \mathrm{~N}_{14} \mathrm{O}_{4} \mathrm{~S}\right)$. Found: $\mathrm{C}, 62.10 ; \mathrm{H}, 4.45 ; \mathrm{N}, 22.16 ; \mathrm{S}, 3.55$; IR ( $\mathrm{KBr}) \mathrm{cm}^{-1} 3300$ ( $\mathrm{NH}$ symm. str.), 3050 (aromatic str.), $1700(\mathrm{C}=\mathrm{O}), 1600$ ( $\mathrm{C}=\mathrm{N}$ str.), ${ }^{1} \mathrm{H}-\mathrm{NMR}$ (DMSO- $\left.d_{6}\right)-\delta$, $11.53(1 \mathrm{H}, \mathrm{s}, \mathrm{NH}), 10.67$ (1H, s, NH), $10.04(1 \mathrm{H}, \mathrm{s}, \mathrm{NH}), 9.44$ $(1 \mathrm{H}, \mathrm{s}, \mathrm{NH}), 8.91(1 \mathrm{H}, \mathrm{s}, \mathrm{NH}), 8.6-6.30$ (18H, m, Ar-H), $4.51\left(2 \mathrm{H}, \mathrm{s}, \mathrm{CH}_{2}\right), 4.03\left(2 \mathrm{H}, \mathrm{s}, \mathrm{CH}_{2}\right), 3.39\left(2 \mathrm{H}, \mathrm{t}, \mathrm{CH}_{2}, \mathrm{~J}=\right.$ $7.2 \mathrm{~Hz}), 2.63\left(2 \mathrm{H}, \mathrm{t}, \mathrm{CH}_{2}, \mathrm{~J}=7.7 \mathrm{~Hz}\right), 2.50\left(4 \mathrm{H}, \mathrm{t}, \mathrm{CH}_{2}, \mathrm{~J}=\right.$ $5.1 \mathrm{~Hz}), 2.33\left(3 \mathrm{H}, \mathrm{s}, \mathrm{CH}_{3}\right), 1.59\left(2 \mathrm{H}, \mathrm{m}, \mathrm{CH}_{2}\right), 1.53(2 \mathrm{H}, \mathrm{m}$, $\mathrm{CH}_{2}$ ).

4-(4-(1-benzyl-4-oxo-2,3,4,5-tetrahydro-1H-pyrido [3,2-b] indol-9-ylamino)-6-(2-(2-oxo-1-(pyrrolidine-1-ylmethyl)indolin-3-ylidene)hydrazinyl)-1,3,5-triazin-2-ylamino)- $N$ (thiazol-2-yl)benzenesulfonamide (7e). Yield $60 \%$; m.p. $313^{\circ} \mathrm{C}$; Anal. Calcd. C, 59.64; H, 4.84; N,21.03; S, 7.41 $\left(\mathrm{C}_{43} \mathrm{H}_{39} \mathrm{~N}_{13} \mathrm{O}_{4} \mathrm{~S}_{2}\right)$. Found: $\mathrm{C}, 59.34 ; \mathrm{H}, 4.34 ; \mathrm{N}, 20.97$; S,7.22; IR ( $\mathrm{KBr}) \mathrm{cm}^{-1} 3300$ (NH symm. str.), 3050 (aromatic str.), $1680(\mathrm{C}=\mathrm{O}), 1622$ (C=N str.), ${ }^{1} \mathrm{H}-\mathrm{NMR}$ (DMSO- $\left.d_{6}\right)-$ $\delta, 11.63(1 \mathrm{H}, \mathrm{s}, \mathrm{NH}), 10.67(1 \mathrm{H}, \mathrm{s}, \mathrm{NH}), 10.04(1 \mathrm{H}, \mathrm{s}, \mathrm{NH})$, $9.44(1 \mathrm{H}, \mathrm{s}, \mathrm{NH}), 8.91(1 \mathrm{H}, \mathrm{s}, \mathrm{NH}), 7.86-6.38(18 \mathrm{H}, \mathrm{m}$, Ar-H), $4.51\left(2 \mathrm{H}, \mathrm{s}, \mathrm{CH}_{2}\right), 4.03\left(2 \mathrm{H}, \mathrm{s}, \mathrm{CH}_{2}\right), 3.39(2 \mathrm{H}, \mathrm{t}$, $\left.\mathrm{CH}_{2} \mathrm{~J}=5.1 \mathrm{~Hz}\right), 2.63\left(2 \mathrm{H}, \mathrm{t}, \mathrm{CH}_{2}, \mathrm{~J}=3.6 \mathrm{~Hz}\right), 2.50(4 \mathrm{H}, \mathrm{t}$, $\left.\mathrm{CH}_{2}, \mathrm{~J}=1.6 \mathrm{~Hz}\right), 1.66\left(4 \mathrm{H}, \mathrm{m}, \mathrm{CH}_{2}\right)$.

4-(4-(1-benzyl-4-oxo-2,3,4,5-tetrahydro-1H-pyrido [3,2-b] indol-9-ylamino)-6-(2-(2-oxo-1-(pyrrolidine-1-ylmethyl)indolin-3-ylidene)hydrazinyl)-1,3,5-triazin-2-ylamino)-N-(6methylpyrazin-2-yl)benzenesulfonamide (7f). Yield 63\%; m.p. $330^{\circ} \mathrm{C}$; Anal. Calcd. C, 61.77; H, 4.73; N, 22.41; S, 7.41 $\left(\mathrm{C}_{45} \mathrm{H}_{42} \mathrm{~N}_{14} \mathrm{O}_{4} \mathrm{~S}\right)$. Found: C, 61.52; H, 4.23; N, 22.22; S, 7.22; IR (KBr) cm 3300 ( $\mathrm{NH}$ symm. str.), 3050 (aromatic str.), 


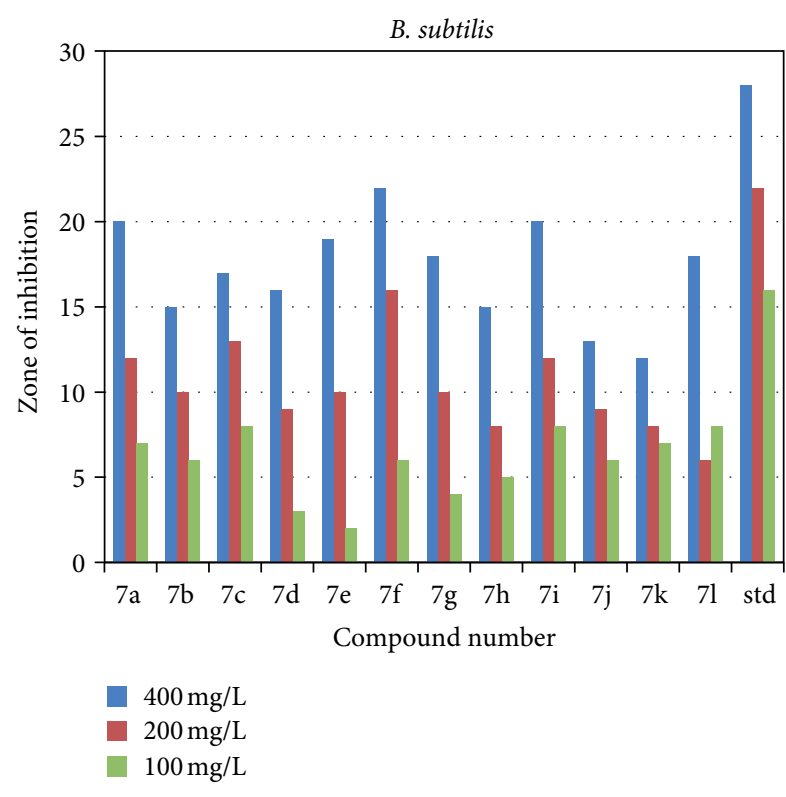

FIgURE 1: Antibacterial activity against B. subtilis.

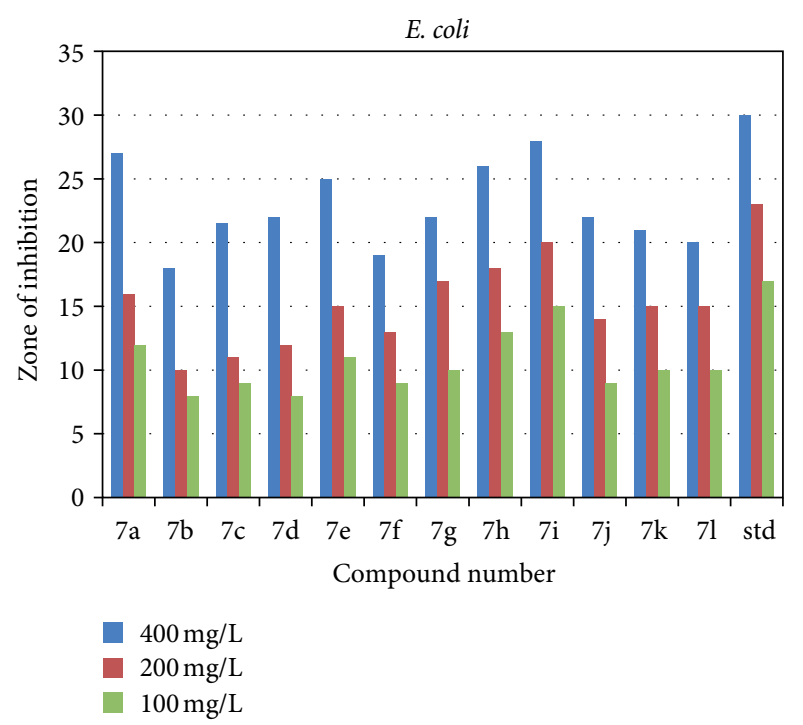

FIgUre 2: Antibacterial activity against E. coli.

$1690(\mathrm{C}=\mathrm{O}), 1625$ (C=N str.), ${ }^{1} \mathrm{H}-\mathrm{NMR}$ (DMSO- $\left.d_{6}\right)-\delta$, $11.53(1 \mathrm{H}, \mathrm{s}, \mathrm{NH}), 10.67(1 \mathrm{H}, \mathrm{s}, \mathrm{NH}), 10.04(1 \mathrm{H}, \mathrm{s}, \mathrm{NH}), 9.44$ $(1 \mathrm{H}, \mathrm{s}, \mathrm{NH}), 8.91(1 \mathrm{H}, \mathrm{s}, \mathrm{NH}), 8.6-6.75(18 \mathrm{H}, \mathrm{m}, \mathrm{Ar}-\mathrm{H})$, $4.51\left(2 \mathrm{H}, \mathrm{s}, \mathrm{CH}_{2}\right), 4.03\left(2 \mathrm{H}, \mathrm{s}, \mathrm{CH}_{2}\right), 3.39\left(2 \mathrm{H}, \mathrm{t}, \mathrm{CH}_{2}, \mathrm{~J}=\right.$ $3.6 \mathrm{~Hz}), 2.63\left(2 \mathrm{H}, \mathrm{t}, \mathrm{CH}_{2}, \mathrm{~J}=2.6 \mathrm{~Hz}\right), 2.51\left(4 \mathrm{H}, \mathrm{t}, \mathrm{CH}_{2}, \mathrm{~J}=\right.$ $2.2 \mathrm{~Hz}), 2.33\left(3 \mathrm{H}, \mathrm{s}, \mathrm{CH}_{3}\right), 1.66\left(4 \mathrm{H}, \mathrm{m}, \mathrm{CH}_{2}\right)$.

4-(4-(1-benzyl-4-oxo-2,3,4,5-tetrahydro-1H-pyrido [3,2-b] indol-9-ylamino)-6-(2-(1-((4-methylpiperazin-1-yl)methyl)-2-oxoindolin-3ylidene)hydrazinyl)-1,3,5-triazin-2ylamino)-N-(6thiazol-2-yl)benzenesulfonamide (7g). Yield 64\%; m.p. $318^{\circ} \mathrm{C}$; Anal. Calcd. C, 59.05; H, 5.02; N, 21.91; S, $7.17\left(\mathrm{C}_{44} \mathrm{H}_{42} \mathrm{~N}_{14} \mathrm{O}_{4} \mathrm{~S}_{2}\right)$. Found: C, 58.45; H, 4.95; N, 22.86; $\mathrm{S}$, 7.07; IR (KBr) cm cm $^{-1} 3300$ (NH symm. str.), 3000 (aromatic str.), 1675 (C=O), 1590 (C=N str.), ${ }^{1} \mathrm{H}-\mathrm{NMR}$ (DMSO- $\left.d_{6}\right)-$ $\delta, 11.63(1 \mathrm{H}, \mathrm{s}, \mathrm{NH}), 10.67(1 \mathrm{H}, \mathrm{s}, \mathrm{NH}), 10.04(1 \mathrm{H}, \mathrm{s}, \mathrm{NH})$, $9.44(1 \mathrm{H}, \mathrm{s}, \mathrm{NH}), 8.91(1 \mathrm{H}, \mathrm{s}, \mathrm{NH}), 7.86-6.30(18 \mathrm{H}, \mathrm{m}$, $\mathrm{Ar}-\mathrm{H}), 4.51\left(2 \mathrm{H}, \mathrm{s}, \mathrm{CH}_{2}\right), 4.03\left(2 \mathrm{H}, \mathrm{s}, \mathrm{CH}_{2}\right), 3.39\left(2 \mathrm{H}, \mathrm{t}, \mathrm{CH}_{2}\right.$, $\mathrm{J}=3.3 \mathrm{~Hz}), 2.53\left(2 \mathrm{H}, \mathrm{t}, \mathrm{CH}_{2}, \mathrm{~J}=1.4 \mathrm{~Hz}\right), 2.35\left(8 \mathrm{H}, \mathrm{t}, \mathrm{CH}_{2}, \mathrm{~J}\right.$ $=2.2 \mathrm{~Hz}), 2.26\left(3 \mathrm{H}, \mathrm{s}, \mathrm{CH}_{3}\right)$.

4-(4-(1-benzyl-4-oxo-2,3,4,5-tetrahydro-1H-pyrido [3,2-b] indol-9-ylamino)-6-(2-(1-((methylpiperazin-1-yl)methyl)-2oxoindolin-3-ylidene)hydrazinyl)-1,3,5-triazin-2-ylamino)$\mathrm{N}$-(6-methylpyrazin-2-yl)benzenesulfonamide (7h). Yield 58\%; m.p. $314^{\circ} \mathrm{C}$; Anal. Calcd. C, 61.12; H, 4.88; N, 23.24; S, $3.55\left(\mathrm{C}_{46} \mathrm{H}_{45} \mathrm{~N}_{15} \mathrm{O}_{4} \mathrm{~S}\right)$. Found: $\mathrm{C}, 61.52 ; \mathrm{H}, 4.77 ; \mathrm{N}, 23.04 ; \mathrm{S}$, 3.51; IR ( $\mathrm{KBr}) \mathrm{cm}^{-1} 3300$ (NH symm. str.), 3005 (aromatic str.), $1710(\mathrm{C}=\mathrm{O}), 1600$ (C=N str.), ${ }^{1} \mathrm{H}-\mathrm{NMR}$ (DMSO- $\left.d_{6}\right)-$ $\delta, 11.53(1 \mathrm{H}, \mathrm{s}, \mathrm{NH}), 10.67(1 \mathrm{H}, \mathrm{s}, \mathrm{NH}), 10.04(1 \mathrm{H}, \mathrm{s}, \mathrm{NH})$, $9.44(1 \mathrm{H}, \mathrm{s}, \mathrm{NH}), 8.91(1 \mathrm{H}, \mathrm{s}, \mathrm{NH}), 8.6(1 \mathrm{H}, \mathrm{s}, \mathrm{Ar}-\mathrm{H}), 8.4$ $(1 \mathrm{H}, \mathrm{s}, \mathrm{Ar}-\mathrm{H}), 7.5-6.2(17 \mathrm{H}, \mathrm{m}, \mathrm{Ar}-\mathrm{H}), 4.51\left(2 \mathrm{H}, \mathrm{s}, \mathrm{CH}_{2}\right)$, $4.03\left(2 \mathrm{H}, \mathrm{s}, \mathrm{CH}_{2}\right), 3.39\left(2 \mathrm{H}, \mathrm{t}, \mathrm{CH}_{2}, \mathrm{~J}=2.2 \mathrm{~Hz}\right), 2.63(2 \mathrm{H}, \mathrm{t}$, $\left.\mathrm{CH}_{2}, \mathrm{~J}=2.6 \mathrm{~Hz}\right), 2.35\left(8 \mathrm{H}, \mathrm{t}, \mathrm{CH}_{2}, \mathrm{~J}=3.6 \mathrm{~Hz}\right), 2.33(3 \mathrm{H}, \mathrm{s}$, $\left.\mathrm{CH}_{3}\right), 2.26\left(3 \mathrm{H}, \mathrm{s}, \mathrm{CH}_{3}\right)$.

4-(4-(1-benzyl-4-oxo-2,3,4,5-tetrahydro-1H-pyrido [3,2-b] indol-9-ylamino)-6-(2-(1-((4-ethylpiperazin-1-yl)methyl)-2oxoindolin-3-ylidene)hydrazinyl)-1,3,5-triazin-2-ylamino)$\mathrm{N}$-(thiazol-2-yl)benzenesulfonamide (7i). Yield 65\%; m.p. $321^{\circ} \mathrm{C}$; Anal. Calcd. C, 59.46; H, 5.16; N, 21.57; S, 7.05 $\left(\mathrm{C}_{45} \mathrm{H}_{44} \mathrm{~N}_{14} \mathrm{O}_{4} \mathrm{~S}_{2}\right)$. Found: C, 59.16; H, 5.06; N, 21.37; S, 6.99; IR ( $\mathrm{KBr}) \mathrm{cm}^{-1} 3300$ (NH symm. str.), 2990 (aromatic str.), $1670(\mathrm{C}=\mathrm{O}), 1595$ ( $\mathrm{C}=\mathrm{N}$ str.), ${ }^{1} \mathrm{H}-\mathrm{NMR}\left(\mathrm{DMSO}-d_{6}\right)-\delta$, $11.63(1 \mathrm{H}, \mathrm{s}, \mathrm{NH}), 10.67(1 \mathrm{H}, \mathrm{s}, \mathrm{NH}), 10.04(1 \mathrm{H}, \mathrm{s}, \mathrm{NH}), 9.44$ $(1 \mathrm{H}, \mathrm{s}, \mathrm{NH}), 8.91(1 \mathrm{H}, \mathrm{s}, \mathrm{NH}), 7.77-6.31(18 \mathrm{H}, \mathrm{m}, \mathrm{Ar}-\mathrm{H})$, $4.51\left(2 \mathrm{H}, \mathrm{s}, \mathrm{CH}_{2}\right), 4.03\left(2 \mathrm{H}, \mathrm{s}, \mathrm{CH}_{2}\right), 3.39\left(2 \mathrm{H}, \mathrm{t}, \mathrm{CH}_{2}, \mathrm{~J}=\right.$ $4.2 \mathrm{~Hz}), 2.53\left(2 \mathrm{H}, \mathrm{t}, \mathrm{CH}_{2}, \mathrm{~J}=3.8 \mathrm{~Hz}\right), 2.35\left(8 \mathrm{H}, \mathrm{t}, \mathrm{CH}_{2}, \mathrm{~J}=\right.$ $2.5 \mathrm{~Hz}), 2.33\left(2 \mathrm{H}, \mathrm{q}, \mathrm{CH}_{2}\right)$.

4-(4-(1-benzyl-4-oxo-2,3,4,5-tetrahydro-1H-pyrido [3,2-b] indol-9-ylamino)-6-(2-(1-((4-ethylpiperazin-1-yl)methyl)-2oxoindolin-3-ylidene)hydrazinyl)-1,3,5-triazin-2-ylamino)$\mathrm{N}$-(6-methylpyrazin-2-yl)benzenesulfonamide (7j). Yield 64\%; m.p. $320^{\circ} \mathrm{C}$; Anal. Calcd. C, 61.49; H, 4.63; N, 22.89; S, 3.49 $\left(\mathrm{C}_{45} \mathrm{H}_{44} \mathrm{~N}_{14} \mathrm{O}_{4} \mathrm{~S}_{2}\right)$. Found: C, 61.19; H, 4.13; N, 22.84; S, 3.43; IR (KBr) cm $\mathrm{cm}^{-1} 3300$ (NH symm. str.), 3005 (aromatic str.), $1690(\mathrm{C}=\mathrm{O}), 1692$ (C=N str.), ${ }^{1} \mathrm{H}-\mathrm{NMR}\left(\mathrm{DMSO}-d_{6}\right)-\delta$, $11.53(1 \mathrm{H}, \mathrm{s}, \mathrm{NH}), 10.67(1 \mathrm{H}, \mathrm{s}, \mathrm{NH}), 10.04(1 \mathrm{H}, \mathrm{s}, \mathrm{NH}), 9.44$ $(1 \mathrm{H}, \mathrm{s}, \mathrm{NH}), 8.91(1 \mathrm{H}, \mathrm{s}, \mathrm{NH}), 8.6-6.38(17 \mathrm{H}, \mathrm{s}, \mathrm{Ar}-\mathrm{H}), 4.51$ $\left(2 \mathrm{H}, \mathrm{s}, \mathrm{CH}_{2}\right), 4.03\left(2 \mathrm{H}, \mathrm{s}, \mathrm{CH}_{2}\right), 3.39\left(2 \mathrm{H}, \mathrm{t}, \mathrm{CH}_{2}, \mathrm{~J}=2.1 \mathrm{~Hz}\right)$, $2.63\left(2 \mathrm{H}, \mathrm{t}, \mathrm{CH}_{2}, \mathrm{~J}=3.1 \mathrm{~Hz}\right), 2.35\left(8 \mathrm{H}, \mathrm{t}, \mathrm{CH}_{2}, \mathrm{~J}=3.3 \mathrm{~Hz}\right)$, $2.33\left(3 \mathrm{H}, \mathrm{s}, \mathrm{CH}_{3}\right), 2.30\left(2 \mathrm{H}, \mathrm{q}, \mathrm{CH}_{2}\right), 1.02\left(3 \mathrm{H}, \mathrm{s}, \mathrm{CH}_{3}\right)$.

4-(4-(1-benzyl-4-oxo-2,3,4,5-tetrahydro-1H-pyrido [3,2-b] indol-9-ylamino)-6-(2-(2-oxo-1-((4-phenylpiperazin-1yl)methyl)indolin-3-ylidene)hydrazinyl)-1,3,5-triazin-2ylamino)- $\mathrm{N}$-(thiazol-2-yl)benzenesulfonamide (7k). Yield 64\%; m.p. $345^{\circ} \mathrm{C}$; Anal. Calcd. C, 61.49; H, 4.54; N, 20.49; $\mathrm{S}$, $6.70\left(\mathrm{C}_{49} \mathrm{H}_{44} \mathrm{~N}_{14} \mathrm{O}_{4} \mathrm{~S}_{2}\right)$. Found: C, 61.09; H, 4.34; N, 20.29; S,7.56; IR (KBr) cm $\mathrm{cm}^{-1} 3300$ (NH symm. str.), 3000 (aromatic str.), $1690(\mathrm{C}=\mathrm{O}), 1595\left(\mathrm{C}=\mathrm{N}\right.$ str.), ${ }^{1} \mathrm{H}-\mathrm{NMR}$ 


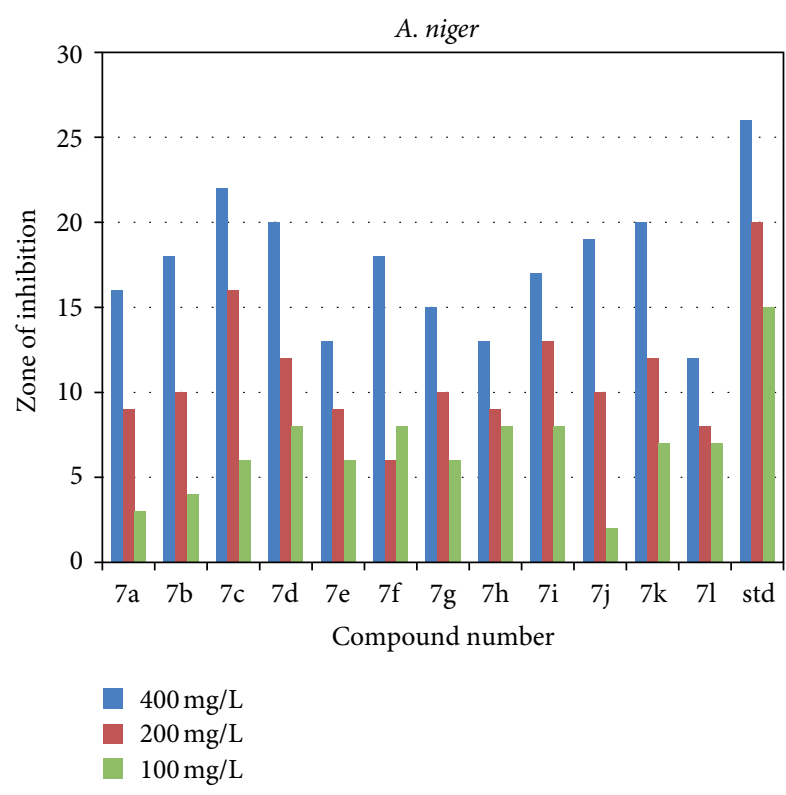

FIgURE 3: Antifungal activity against A. niger.

$\left(\mathrm{DMSO}-d_{6}\right)-\delta, 11.53(1 \mathrm{H}, \mathrm{s}, \mathrm{NH}), 10.67(1 \mathrm{H}, \mathrm{s}, \mathrm{NH}), 10.04$ (1H, s, NH), $9.44(1 \mathrm{H}, \mathrm{s}, \mathrm{NH}), 8.91(1 \mathrm{H}, \mathrm{s}, \mathrm{NH}), 7.86-6.79$ $(22 \mathrm{H}, \mathrm{m}, \mathrm{Ar}-\mathrm{H}), 4.51\left(2 \mathrm{H}, \mathrm{s}, \mathrm{CH}_{2}\right), 4.03\left(2 \mathrm{H}, \mathrm{s}, \mathrm{CH}_{2}\right), 3.44$ $\left(4 \mathrm{H}, \mathrm{t}, \mathrm{CH}_{2}, \mathrm{~J}=1.6 \mathrm{~Hz}\right), 3.39\left(2 \mathrm{H}, \mathrm{t}, \mathrm{CH}_{2}, \mathrm{~J}=1.9 \mathrm{~Hz}\right), 2.63$ $\left(2 \mathrm{H}, \mathrm{t}, \mathrm{CH}_{2}, \mathrm{~J}=1.9 \mathrm{~Hz}\right), 2.48\left(4 \mathrm{H}, \mathrm{t}, \mathrm{CH}_{2}, \mathrm{~J}=2.6 \mathrm{~Hz}\right)$.

4-(4-(1-benzyl-4-oxo-2,3,4,5-tetrahydro-1H-pyrido [3,2-b] indol-9-ylamino)-6-(2-(2-oxo-1-((4-phenylpiperazin-1yl)methyl)indolin-3-ylidene)hydrazinyl)-1,3,5-triazin-2ylamino)-N-(6-methylpyrazin-2-yl)benzenesulfonamide (71). Yield 65\%; m.p. $335^{\circ} \mathrm{C}$; Anal. Calcd. C, 63.41; H, 4.90; N, 21.75; S, $3.32\left(\mathrm{C}_{51} \mathrm{H}_{47} \mathrm{~N}_{15} \mathrm{O}_{4} \mathrm{~S}\right)$. Found: $\mathrm{C}$ 63.01; $\mathrm{H}, 4.75 ; \mathrm{N}$, 21.55; S, 3.02; IR (KBr) cm $\mathrm{cm}^{-1} 3300$ (NH symm. str.), 3010 (aromatic str.), $1680(\mathrm{C}=\mathrm{O}), 1610$ (C=N str.), ${ }^{1} \mathrm{H}-\mathrm{NMR}$ $\left(\mathrm{DMSO}-d_{6}\right)-\delta, 11.53(1 \mathrm{H}, \mathrm{s}, \mathrm{NH}), 10.67(1 \mathrm{H}, \mathrm{s}, \mathrm{NH}), 10.04$ $(1 \mathrm{H}, \mathrm{s}, \mathrm{NH}), 9.44(1 \mathrm{H}, \mathrm{s}, \mathrm{NH}), 8.91(1 \mathrm{H}, \mathrm{s}, \mathrm{NH}), 8.4-6.28$ $(23 \mathrm{H}, \mathrm{m}, \mathrm{Ar}-\mathrm{H}), 4.51\left(2 \mathrm{H}, \mathrm{s}, \mathrm{CH}_{2}\right), 4.03\left(2 \mathrm{H}, \mathrm{s}, \mathrm{CH}_{2}\right)$, 3.44(4H, t, $\left.\mathrm{CH}_{2}, \mathrm{~J}=7.2 \mathrm{~Hz}\right), 3.39\left(2 \mathrm{H}, \mathrm{t}, \mathrm{CH}_{2}, \mathrm{~J}=2.2 \mathrm{~Hz}\right)$, $2.63\left(2 \mathrm{H}, \mathrm{t}, \mathrm{CH}_{2}, \mathrm{~J}=5.9 \mathrm{~Hz}\right), 2.48\left(4 \mathrm{H}, \mathrm{t}, \mathrm{CH}_{2}, \mathrm{~J}=6.6 \mathrm{~Hz}\right)$, $2.33\left(3 \mathrm{H}, \mathrm{s}, \mathrm{CH}_{3}\right)$.

\section{Biological Assay}

Among all of the synthesized compounds, compounds 7(a-l) were screened for antimicrobial activity by disc diffusion method [18]. The test compounds $7(\mathbf{a}-\mathbf{l})$, in measured quantities, were dissolved in dimethyl formamide (DMF) to get the final concentrations $400 \mathrm{mg} / \mathrm{L}, 200 \mathrm{mg} / \mathrm{L}, 100 \mathrm{mg} / \mathrm{L}$ respectively. The bacterial $(24 \mathrm{~h})$ and fungal $(48 \mathrm{~h})$ cultures from the slants were diluted with sterile water and mixed thoroughly to prepare a clear homogeneous suspension. These suspensions were spread on solidified agar (NA-nutrient agar-for bacteria and PDA — potato dextrose agar for fungi)

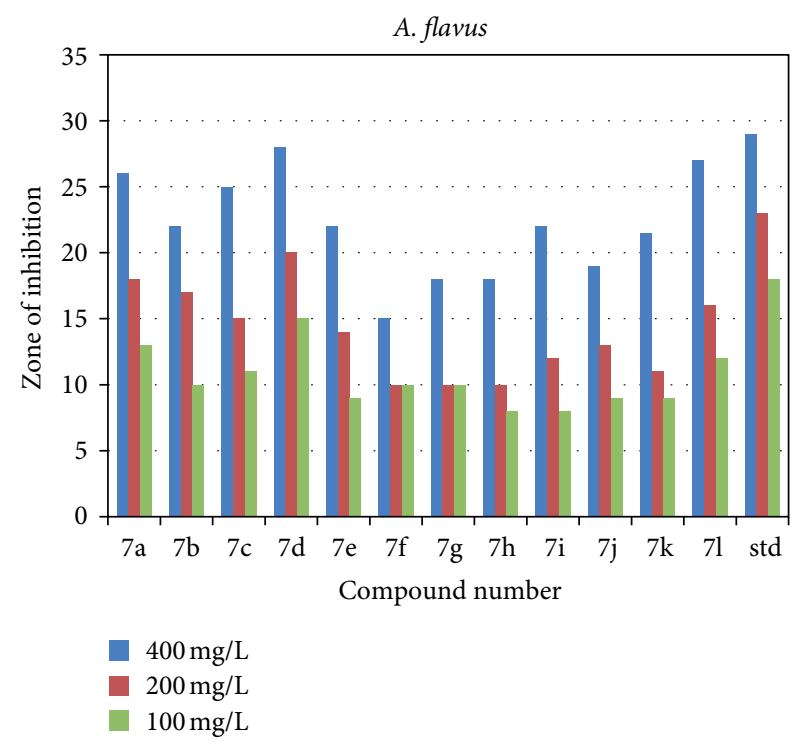

FIGURE 4: Antifungal activity against $A$. flavus.

medium. The filter paper disks [18] prepared by only DMF (as a negative control) and with solutions of different concentrations of test compounds $7(\mathbf{a}-\mathbf{l})$ as well as standard compounds (ciprofloxacin and fluconazole as positive control) were carefully placed over the spread cultures and incubated at $37^{\circ} \mathrm{C}$ for $24 \mathrm{hr}$ for bacteria and at $28-30^{\circ} \mathrm{C}$ for $48 \mathrm{hr}$ for fungi. After the incubation period, the plates were examined for the zone of inhibition. The diameter for the zones of inhibition were measured including the diameter of disk also. All determinations were made in triplicate for each of the compound and the average value was taken. The antibacterial and antifungal activity was evaluated against $E$. coli, B. subtilis, A. niger, and A. flavus against the standard drugs ciprofloxacin for bacteria and fluconazole for fungi. The outcome of this study is presented in Table 1 and in Figures $1-4$.

\section{Results and Discussion}

The synthetic approach which allowed the incorporation of the aforementioned bioactive heterocyclic systems on to the 2, 4, and 6 positions of s-triazine molecule was confined to three series outlined in Schemes 1, 2, and 3. The first series was derived from isatin which afforded its hydrazone from the reaction with hydrazine hydrate. Scheme 1 shows the synthetic strategy applied to obtain $3(\mathbf{a}-\mathbf{f})$ by replacing one of the chlorine atoms of s-triazine (1) with isatinyl-3-hydrazone Mannich's bases 2(a-f), by conducting the reaction in Dry $\mathrm{THF} / \mathrm{K}_{2} \mathrm{CO}_{3}$ at $0-5^{\circ} \mathrm{C}$ for $2-3 \mathrm{hrs}$. Replacement of chlorine atoms of s-triazine is a temperature dependent nucleophilic substitution reaction [19]. While the second series was derived by the replacement of one of the chlorine atoms of $\mathbf{3}(\mathbf{a}-\mathbf{f})$ with two established sulpha drugs $\mathbf{4}(\mathbf{a}, \mathbf{b})$, consisting of sulphathiazole and sulphamerazine respectively, to yield of $5(\mathrm{a}-\mathbf{l})$ by conducting the reaction in $\mathrm{THF} / \mathrm{K}_{2} \mathrm{CO}_{3}$ at $30-35^{\circ} \mathrm{C}$ for $2-3 \mathrm{hrs}$. 


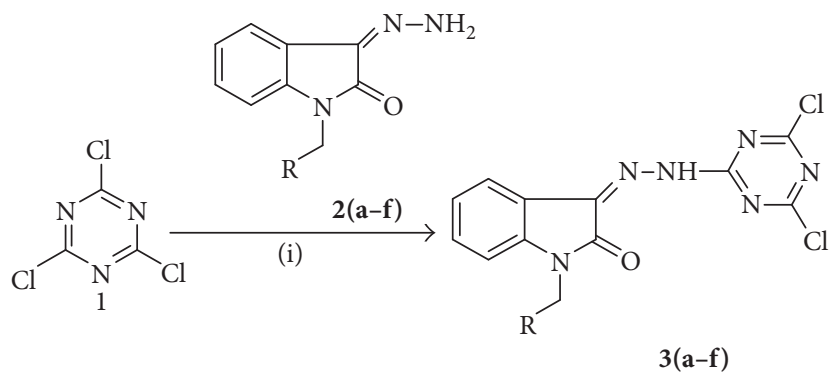

Scheme 1: Monosubstitution reaction (i) Dry THF, $0-5^{\circ} \mathrm{C}, \mathrm{K}_{2} \mathrm{CO}_{3} . \mathbf{3 a}, \mathrm{R}=$ morpholinyl, $\mathbf{3 b}, \mathrm{R}=$ piperidinyl, $\mathbf{3} \mathbf{c}, \mathrm{R}=$ pyrrolidinyl, $\mathbf{3 d}$, $\mathrm{R}=$ $\mathrm{N}$-methyl piperazinyl, $\mathbf{3 e}, \mathrm{R}=\mathrm{N}$-ethyl piperazinyl, 3f, $\mathrm{R}=\mathrm{N}$-phenyl piperazinyl.

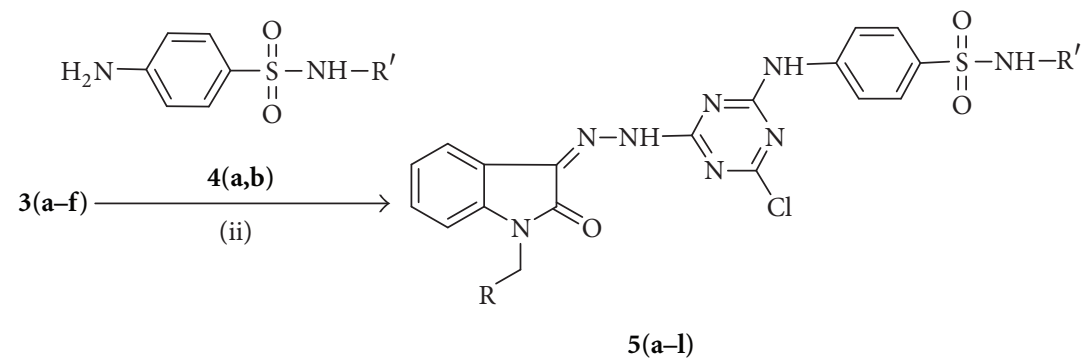

SCHEme 2: Disubstitution (ii) dry THF, 30-35 ${ }^{\circ} \mathrm{C}, \mathrm{K}_{2} \mathrm{CO}_{3} . \mathbf{5} \mathbf{a}, \mathrm{R}=$ morpholinyl, $\mathrm{R}^{\prime}=$ thiazolyl, $\mathbf{5 b}, \mathrm{R}=$ morpholinyl, $\mathrm{R}^{\prime}=$ methyl pyrimidinyl, $\mathbf{5 c}, \mathrm{R}=$ piperidinyl, $\mathrm{R}^{\prime}=$ thiazolyl, $5 \mathrm{~d}, \mathrm{R}=$ piperidinyl, $\mathrm{R}^{\prime}=$ methyl pyrimidinyl, $\mathbf{5 e}, \mathrm{R}=$ pyrrolidinyl, $\mathrm{R}^{\prime}=$ thiazolyl, $5 \mathrm{f}, \mathrm{R}=$ pyrrolidinyl, $\mathrm{R}^{\prime}$ $=$ methyl pyrimidinyl, $\mathbf{5} \mathbf{g}, \mathrm{R}=\mathrm{N}$-methyl piperazinyl, $\mathrm{R}^{\prime}=$ thiazolyl, $\mathbf{5} \mathbf{h}, \mathrm{R}=\mathrm{N}$-methyl piperazinyl, $\mathrm{R}^{\prime}=$ methyl pyrimidinyl, $\mathbf{5 i}, \mathrm{R}=\mathrm{N}$-ethyl piperazinyl, $\mathrm{R}^{\prime}=$ thiazolyl, 5j, $\mathrm{R}=\mathrm{N}$-ethyl piperazinyl, $\mathrm{R}^{\prime}=$ methyl pyrimidinyl, 5k, $\mathrm{R}=\mathrm{N}$-phenyl piperazinyl $\mathrm{R}^{\prime}=$ thiazolyl, 5l, $\mathrm{R}=\mathrm{N}$-phenyl piperazinyl, $\mathrm{R}^{\prime}=$ methyl pyrimidinyl.<smiles>Nc1ccc2[nH]c3c(c2c1)N(Cc1ccccc1)CCC3=O</smiles>

$5(\mathrm{a}-\mathrm{l})$

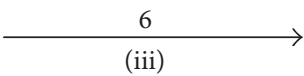

(iii)<smiles>[R]CN1C(=O)/C(=N\Nc2nc(Nc3ccc(S(=O)(=O)N[R])cc3)nc(Nc3cccc4[nH]c5c(c34)N(Cc3ccccc3)CCC5=O)n2)c2ccccc21</smiles>

$7(\mathbf{a}-\mathbf{l})$

Scheme 3: Trisubstitution (iii) dry THF, reflux $\left(60-65^{\circ} \mathrm{C}\right), \mathrm{K}_{2} \mathrm{CO}_{3}$.<smiles></smiles>

8

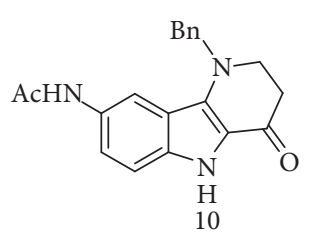

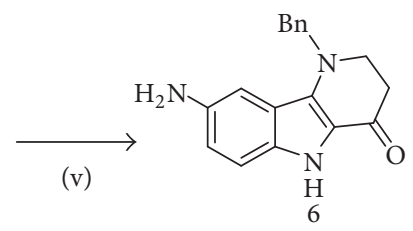

Scheme 4: Synthesis of N-benzyl-8-amino-azacarbazole-4-one (4) (a) Japp-Klingemann-sodium acetate trihydrate, methanol, water (b) Fischer-indolization-acetic acid, $\mathrm{HCl}$ (5) hydrolysis, Dil. $\mathrm{HCl}$. 
TABle 1: Antibacterial and antifungal activities of synthesized compounds using disc diffusion method.

\begin{tabular}{|c|c|c|c|c|c|c|c|c|c|c|c|c|}
\hline \multirow{3}{*}{ S. number } & \multicolumn{12}{|c|}{ Zone of inhibition $(\mathrm{mm})$} \\
\hline & & B. subtilis & & & E. coli & & & A. niger & & & A. flavus & \\
\hline & $400 \mathrm{mg} / \mathrm{L}$ & $200 \mathrm{mg} / \mathrm{L}$ & $100 \mathrm{mg} / \mathrm{L}$ & $400 \mathrm{mg} / \mathrm{L}$ & $200 \mathrm{mg} / \mathrm{L}$ & $100 \mathrm{mg} / \mathrm{L}$ & $400 \mathrm{mg} / \mathrm{L}$ & $200 \mathrm{mg} / \mathrm{L}$ & $100 \mathrm{mg} / \mathrm{L}$ & $400 \mathrm{mg} / \mathrm{L}$ & $200 \mathrm{mg} / \mathrm{L}$ & $100 \mathrm{mg} / \mathrm{L}$ \\
\hline $7 \mathrm{a}$ & 20 & 12 & 7 & 27 & 16 & 12 & 16 & 9 & 3 & 26 & 18 & 13 \\
\hline $7 \mathrm{~b}$ & 15 & 10 & 6 & 18 & 10 & 8 & 18 & 10 & 4 & 22 & 17 & 10 \\
\hline $7 \mathrm{c}$ & 17 & 13 & 8 & 21.5 & 11 & 9 & 22 & 16 & 6 & 25 & 15 & 11 \\
\hline $7 \mathrm{~d}$ & 16 & 9 & 3 & 22 & 12 & 8 & 20 & 12 & 8 & 28 & 20 & 15 \\
\hline $7 e$ & 19 & 10 & 2 & 25 & 15 & 11 & 13 & 9 & 6 & 22 & 14 & 9 \\
\hline $7 f$ & 22 & 16 & 6 & 19 & 13 & 9 & 18 & 6 & 8 & 15 & 10 & 10 \\
\hline $7 \mathrm{~g}$ & 18 & 10 & 4 & 22 & 17 & 10 & 15 & 10 & 6 & 18 & 10 & 10 \\
\hline $7 \mathrm{~h}$ & 15 & 8 & 5 & 26 & 18 & 13 & 13 & 9 & 8 & 18 & 10 & 8 \\
\hline $7 \mathrm{i}$ & 20 & 12 & 8 & 28 & 20 & 15 & 17 & 13 & 8 & 22 & 12 & 8 \\
\hline $7 j$ & 13 & 9 & 6 & 22 & 14 & 9 & 19 & 10 & 2 & 19 & 13 & 9 \\
\hline $7 \mathrm{k}$ & 12 & 8 & 7 & 21 & 15 & 10 & 20 & 12 & 7 & 21.5 & 11 & 9 \\
\hline 71 & 18 & 6 & 8 & 20 & 15 & 10 & 12 & 8 & 7 & 27 & 16 & 12 \\
\hline $\begin{array}{l}\text { Cipro. } \\
\text { (Std.) }\end{array}$ & 28 & 22 & 16 & 30 & 23 & 17 & - & - & - & - & - & - \\
\hline $\begin{array}{l}\text { Fluco. } \\
\text { (Std.) }\end{array}$ & - & - & - & - & - & - & 26 & 20 & 15 & 29 & 23 & 18 \\
\hline
\end{tabular}

The third series which consisted of the target compounds $7(\mathbf{a}-\mathbf{l})$, was derived by replacement of the last chlorine atom of $5(\mathrm{a}-\mathrm{l})$ with $\mathrm{N}$-benzyl-8-amino-azacarbazole-4-one derivative (6). The reaction was carried out in $\mathrm{THF} / \mathrm{K}_{2} \mathrm{CO}_{3}$ medium at $60-65^{\circ} \mathrm{C}$ for 6 hrs. as shown in Scheme 3.

Compound, N-benzyl-8-amino-azacarbazole-4-one (6) required in the preparation of the target compounds, $7(\mathbf{a}-\mathbf{l})$, was synthesized from $\mathrm{p}$-acetamido benzene diazonium chloride (8) following the strategy shown in Scheme 4. The reaction of 8 when allowed to take place with 3-hydroxy methylidene-N-benzyl-4-piperidone (9) under the conditions of Japp-Klingeman [20] reaction afforded the corresponding hydrazone 10, from which 6 resulted on Fischer indolization with Kent's reagent $[\mathrm{AcOH}: \mathrm{HCl}, 4: 1, \mathrm{v} / \mathrm{v}]$ followed by subsequent hydrolysis with acid.

The antibacterial and antifungal activity was evaluated against E. coli, B. subtilis, A. niger, and A. flavus against the standard drugs ciprofloxacin for bacteria and fluconazole for fungi. The zone of inhibition was measured in $\mathrm{mm}$, on three different concentrations $400 \mathrm{mg} / \mathrm{L}, 200 \mathrm{mg} / \mathrm{L}$, $100 \mathrm{mg} / \mathrm{L}$. Maximum inhibitory activity was observed for $400 \mathrm{mg} / \mathrm{L}$ and compounds showed their effect in a dosedependent manner. The compound $7 \mathbf{f}$ was found to be most potent against $B$. subtilis, while the compound $7 \mathbf{a}$ and $7 \mathbf{i}$ these were found to be equipotent remaining compounds also showed the promising antibacterial activity. The compounds $7 \mathbf{a}, 7 \mathbf{i}$ these were most potent against $E$. coli while the compound $7 \mathbf{e}$ and $7 \mathbf{h}$ these were equipotent against the same strain as shown in Figures 1 and 2. The compounds $7 \mathbf{c}$ and $7 d$, these showed promising activity against $A$. niger and $A$. flavus respectively, while the remaining compounds also showed promising inhibitory activity as shown in Figures 3 and 4. The morpholine, pyrrole ring, and thiazole ring were found more potent in their inhibitory effect on growth of both bacterial and fungal strains which indicates the positive modulator effect of electro negativity on activity in these molecules.

\section{Conclusion}

We developed novel trisubstitute triazine derivatives containing, N-Mannich bases of isatin-3-hydrazones, established sulpha drugs and N-benzyl-4-oxo azacarbazole derivatives using nucleophilic substitution reaction, Japp-Klingmann, Fischer-indolization, and other reactions. Furthermore, it was found that the morpholine, pyrrole ring, and thiazole ring derivatives $7 \mathbf{a}, 7 \mathbf{i}$, and $7 \mathbf{h}$ were more potent in their inhibitory effect on growth of both bacterial and fungal strains which indicates the positive modulator effect of electronegativity on activity in these molecules. Their further quantitative structure relationship (QSAR) studies are currently under investigation.

\section{Acknowledgments}

Authors are grateful to the director, CDRI, Lucknow and Dr. Anees A. siddiqui, Jamiya Humdard University Delhi, for providing the ${ }^{1} \mathrm{H}-\mathrm{NMR}$ data of the compounds and to the Department of Bioscience and Biotechnology for providing the facilities to conduct antimicrobial activity of the compounds. Authors are highly thankful for financial support provided by Department of Science and Technology (DST), New Delhi for the project, "Banasthali Center for Education and Research in Basic Sciences" under their CURIE (Consolidation of University Research for Innovation and Excellence in women Universities) Programme.

\section{References}

[1] J. P. Raval, A. R. Rai, and N. H. Patel, "Synthesis and in vitro antimicrobial activity of $\mathrm{N}^{\prime}$-(4-(arylamino)-6-(pyridin-2ylamino)-1,3,5-triazin-2-yl)benzohydrazide," International Journal of ChemTech Research, vol. 3, no. 1, pp. 616-620, 2009. 
[2] S. Nishigaki, F. Yoneda, and K. Morinaga, "Synthetic antibacterials. I. Nitrofurylvinyl-s-triazine derivatives," Journal of Medicinal Chemistry, vol. 12, no. 1, pp. 39-42, 1969.

[3] L. D. S. Yadav, K. N. Shukla, R. Dwivedi, and H. Singh, "Synthesis of new 1,3,4-oxadiazolo [3,2-a]-s-triazine-5,7-dithiones and the dithionone analogues as potential antifungal agents," Indian Journal of Pharmaceutical Sciences, vol. 54, no. 1, pp. 33-37, 1992.

[4] D. H. Mahajan, C. Pannecouque, E. de Clercq, and K. H. Chikhalia, "Synthesis and studies of new 2-(coumarin-4yloxy)-4,6-(substituted)-s-triazine derivatives as potential antiHIV agents," Archiv der Pharmazie, vol. 342, no. 5, pp. 281-290, 2009.

[5] S. C. Yaguchi, Y. Chin Fukui, I. Koshimizu et al., "Antitumor activity of ZSTK474, a new phosphatidylinositol 3-kinase inhibitor," Journal of the National Cancer Institute, vol. 98, no. 8, pp. 545-556, 2006.

[6] V. K. Pandey, S. Tusi, Z. Tusi, M. Joshi, and S. Bajpai, "Synthesis and biological activity of substituted 2,4,6-s-triazines," Acta Pharmaceutica, vol. 54, no. 1, pp. 1-12, 2004.

[7] A. Agrawal, K. Shrivastav, S. K. Puri, and M. S. Chauhan, "Syntheses of 2,4,6-trisubstituted triazines as antimalarial agents," Bioorganic and Medicinal Chemistry Letters, vol. 15, no. 3, pp. 531-533, 2005.

[8] G. S. Singh, T. Singh, and R. Lakhan, "Synthesis, 13C NMR and anticonvulsant activity of new isatin-based spiroazetidinones," Indian Journal of Chemistry B, vol. 36, no. 10, pp. 951-954, 1997.

[9] F. D. Popp and H. Pajouhesh, "Potential anticonvulsant. IV: Condensation of isatin with benzoylacetone and isopropyl/ methyl ketones," Journal of Pharmaceutical Sciences, vol. 71, pp. 1052-1054, 1982.

[10] S. K. Bhattacharya and A. Chakrabarti, "Dose-related proconvulsant and anticonvulsant activity of isatin, a putative biological factor, in rats," Indian Journal of Experimental Biology, vol. 36, no. 1, pp. 118-121, 1998.

[11] K. V. Sujith, J. N. Rao, P. Shetty, and B. Kalluraya, "Regioselective reaction: synthesis and pharmacological study of Mannich bases containing ibuprofen moiety," European Journal of Medicinal Chemistry, vol. 44, no. 9, pp. 3697-3702, 2009.

[12] C. T. Supuran, A. Casini, and A. Scozzafava, "Protease inhibitors of the sulfonamide type: anticancer, antiinflammatory, and antiviral agents," Medicinal Research Reviews, vol. 23, no. 5, pp. 535-558, 2003.

[13] A. Scozzafava, T. Owa, A. Mastrolorenzo, and C. T. Supuran, "Anticancer and antiviral sulfonamides," Current Medicinal Chemistry, vol. 10, no. 11, pp. 925-953, 2003.

[14] J. Y. Winum, A. Scozzafava, J. L. Montero, and C. T. Supuran, "Therapeutic potential of sulfamides as enzymes inhibitors," Medicinal Research Reviews, vol. 26, no. 6, pp. 767-792, 2006.

[15] K. Isik and K. Özdemir, "Antimicrobial activity screening of some sulfonamide derivatives on some Nocardia species and isolates," Microbiological Research, vol. 164, no. 1, pp. 49-58, 2009.

[16] M. R. Burns, S. A. Jenkins, M. R. Kimbrell et al., "Polycationic sulfonamides for the sequestration of endotoxin," Journal of Medicinal Chemistry, vol. 50, no. 4, pp. 877-888, 2007.

[17] L. M. Werbel, M. Angelo, D. W. Fry, and D. F. Worth, "Basically substituted ellipticine analogues as potential antitumor agents," Journal of Medicinal Chemistry, vol. 29, no. 7, pp. 1321-1322, 1986.
[18] R. D. Biljana, S. R. Niko, S. D. Vidoslav, R. D. Vukicević, and R. M. Palić, "Synthesis and antimicrobial activity of new 4heteroarylamino coumarin derivatives containing nitrogen and sulfur as heteroatoms," Molecules, vol. 15, no. 4, pp. 2246-2256, 2010.

[19] A. M. Carlos, M. T. Nuno, and A. R. Andreia, "Synthesis of 2,4,6Tri-substituted-1,3,5-Triazines," Molecules, vol. 11, pp. 81-102, 2006.

[20] F. R. Japp and F. Klingemann, "Ueber die constitution einiger sogenannten gemischten azoverbindungen," Liebigs Annalen der Chemie, vol. 247, no. 2, pp. 190-225, 1888. 

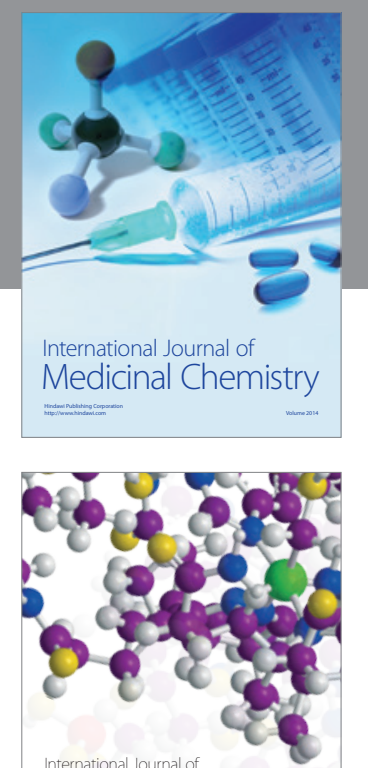

\section{Carbohydrate} Chemistry

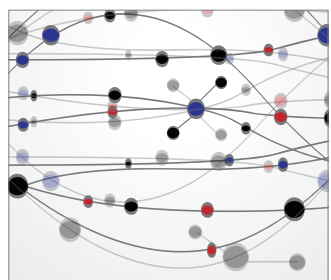

The Scientific World Journal
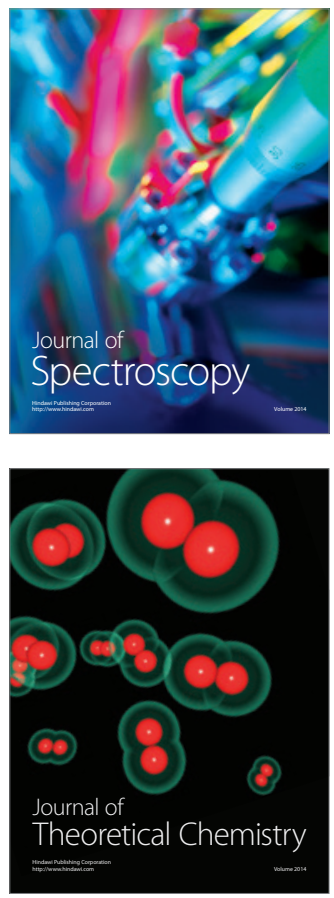
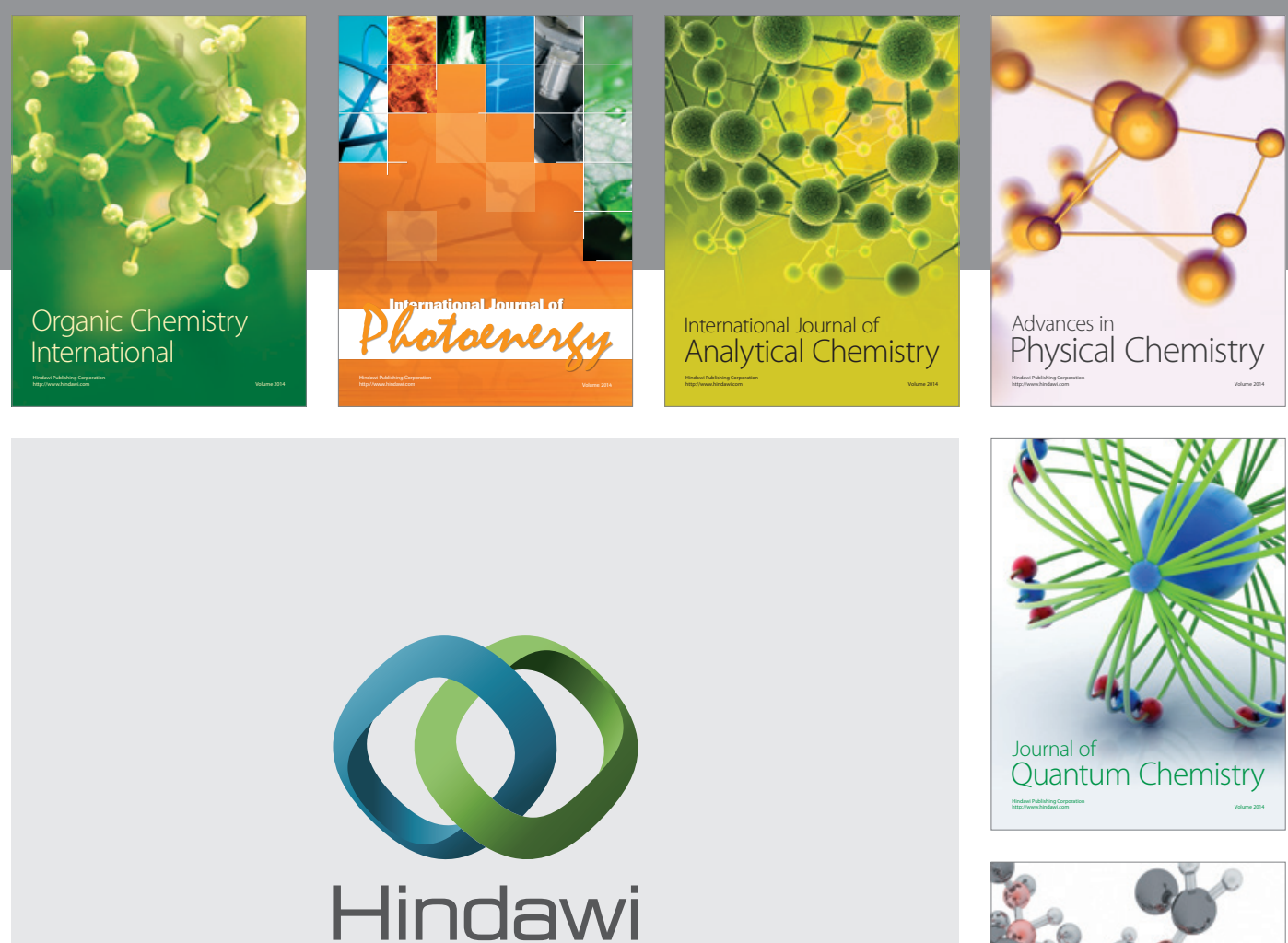

Submit your manuscripts at

http://www.hindawi.com

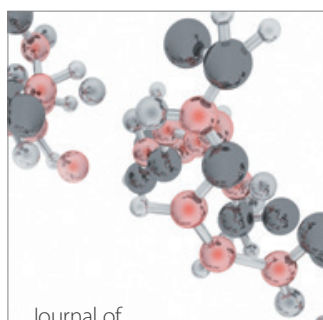

Analytical Methods

in Chemistry

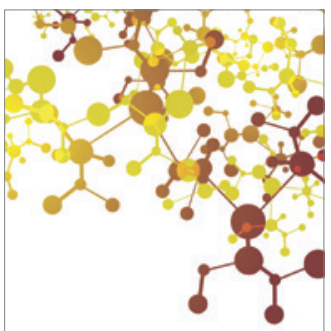

Journal of

Applied Chemistry

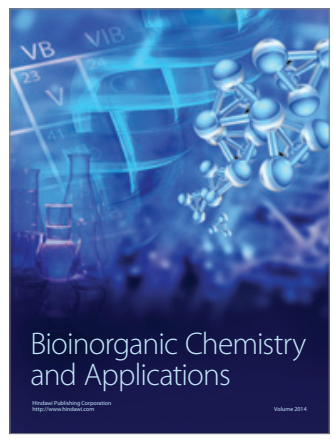

Inorganic Chemistry
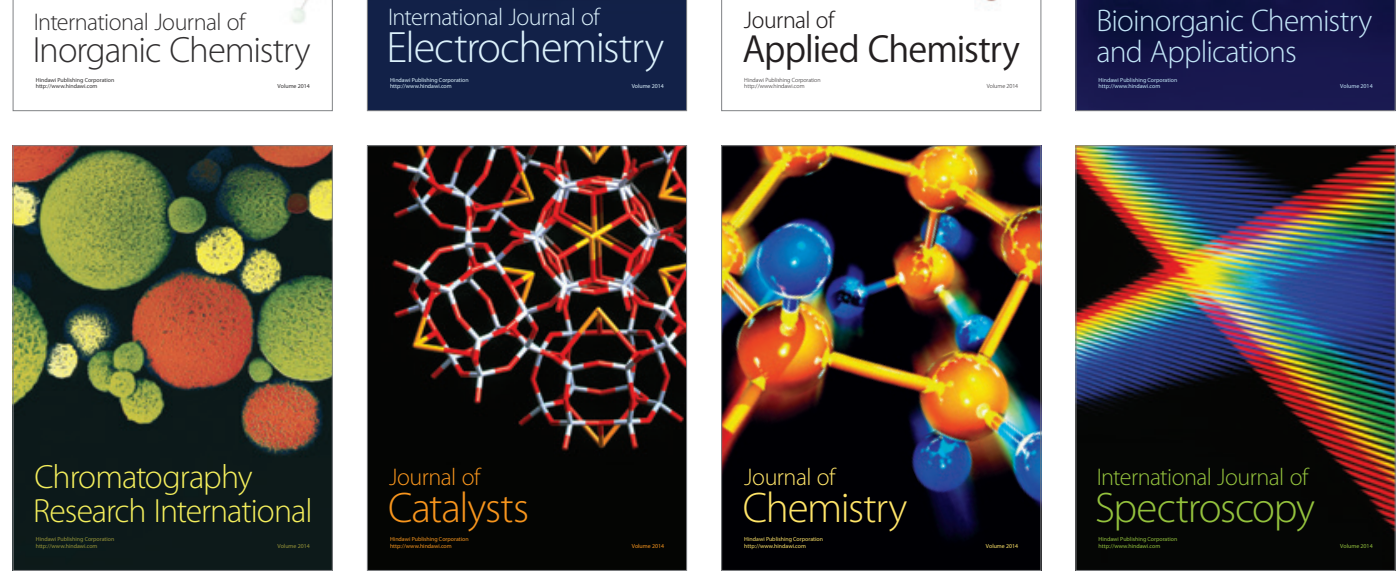